PUPT-2253

SU-ITP-07/25

ITEP-TH-79/07

\title{
On Normal Modes of a Warped Throat
}

\author{
Marcus K. Benna ${ }^{a}$, Anatoly Dymarsky ${ }^{b}$ Igor R. Klebanov $^{a, c}$ and Alexander Solovyov ${ }^{a, d}$ \\ a Department of Physics, Princeton University, Princeton, NJ 08544 \\ ${ }^{b}$ Stanford Institute for Theoretical Physics, Stanford, CA 94305 \\ ${ }^{c}$ Princeton Center for Theoretical Physics, Princeton University, Princeton, NJ 08544 \\ ${ }^{d}$ Bogolyubov Institute for Theoretical Physics, Kiev 03680, Ukraine
}

\begin{abstract}
As shown in arXiv:hep-th/0405282, the warped deformed conifold has two bosonic massless modes, a pseudoscalar and a scalar, that are dual to the phase and the modulus of the baryonic condensates in the cascading gauge theory. We reconsider the scalar mode sector, mixing fluctuations of the NS-NS 2-form and the metric, and include non-zero 4-d momentum $k_{\mu}$. The resulting pair of coupled equations produce a discrete spectrum of $m_{4}^{2}=-k_{\mu}^{2}$ which is interpreted as the spectrum of $J^{P C}=0^{+-}$glueballs in the gauge theory. Similarly, we derive the spectrum of certain pseudoscalar glueballs with $J^{P C}=0^{--}$, which originate from the decoupled fluctuations of the RR 2-form. We argue that each of the massive scalar or pseudoscalar modes we find belongs to a 4-d massive axial vector or vector supermultiplet. We also discuss our results in the context of a finite length throat embedded into a type IIB flux compactification.
\end{abstract}




\section{Introduction}

Duality between the cascading $S U(k(M+1)) \times S U(k M)$ gauge theory and type IIB strings on the warped deformed conifold [1] provides a rich yet solvable example of gauge/string correspondence [2, 3, 4]. For earlier work leading up to this duality, see [5, 6, 7, 8], and for reviews [9, 10]. This background demonstrates in a geometrical language such features of the $S U(M)$ supersymmetric gluodynamics as color confinement and the breaking of the $Z_{2 M}$ chiral R-symmetry down to $Z_{2}$ via gluino condensation [1]. In fact, it has been argued [1] that by reducing the continuous parameter $g_{s} M$ one can interpolate between the cascading theory solvable in the supergravity limit and $\mathcal{N}=1$ supersymmetric $S U(M)$ gauge theory.

The problem of finding the spectra of bound states at large $g_{s} M$ can be mapped to finding normalizable fluctuations around the supergravity background. This problem is complicated by the presence of 3-form and 5-form fluxes, but some results on the spectra are already available in the literature [11, 12, 13, 14, 15, 16, 17]. A particularly impressive effort was made by Berg, Haack and Mück (BHM) who used a generalized PT ansatz [18] to derive and numerically solve a system of seven coupled scalar equations [14, 15]. Each of the resulting glueballs is even under the charge conjugation $Z_{2}$ symmetry preserved by the KS solution (this symmetry was called the $\mathcal{I}$-symmetry in [13]), and therefore has $J^{P C}=0^{++}$. The present paper will study three other families of glueballs, which are odd under the $\mathcal{I}$-symmetry. Two of them originate from a pair of coupled scalar equations, generalizing the zero momentum case studied in [13], and have $J^{P C}=0^{+-}$. The third, pseudoscalar family arises from a decoupled fluctuation of the RR two-form $C_{2}$ and has $J^{P C}=0^{--}$.

An important aspect of the low-energy dynamics is that the baryonic $U(1)_{B}$ symmetry is broken spontaneously by the condensates of baryonic operators $\mathcal{A}$ and $\mathcal{B}$. This phenomenon, anticipated in the cascading gauge theory in [1, 19], was later demonstrated on the supergravity side where the fluctuations corresponding to the pseudoscalar Goldstone boson and its scalar superpartner [13], as well as the fermionic superpartner [16], were identified. Furthermore, finite deformations along the scalar direction give rise to a continuous family of supergravity solutions [20, 21, 22] dual to the baryonic branch, $\mathcal{A B}=$ const, of the gauge theory moduli space.

The main purpose of the present paper is to obtain a deeper understanding of the GHK scalar fluctuations [13] and their radial excitations. Our motivation is two-fold. On the one hand, we seek an improved understanding of the glueball spectra and their supermultiplet structure. On the other, we would like to shed new light on the normal modes of the warped deformed conifold throat embedded into a string compactification, which has played a role in models of moduli stabilization [23] and D-brane inflation [24, 25]. In such inflation models, the reheating of the universe involves emission of modes localized near the bottom of the throat, which are dual to glueballs in the gauge theory [26, 27, 28].

This paper is structured as follows. In section 2 we construct a generalization of the ansatz for the NSNS 2-form and metric perturbations that allows us to study radial excitations of the GHK scalar mode. We derive a system of coupled radial equations and determine their spectrum 
(the details of the numerical treatment are presented in Appendix (D). In section 3 we show that a similar ansatz for the RR 2-form perturbation decouples from the metric giving rise to a single decoupled equation for pseudoscalar glueballs. In section 4 we argue that the scalar glueballs we find belong to massive axial vector multiplets, and the pseudoscalar glueballs belong to massive vector multiplets. Agreement of the corresponding equations is explicitly demonstrated in the large radius (KT) limit. In section 5 we give a perturbative treatment of the coupled equations for small mass that allows us to study the scalar mass in models where the length of the throat is finite. Review of the supergravity equations and of the warped deformed conifold, as well as some technical details, are delegated to the Appendices.

\section{Radial Excitations of the GHK scalar}

The ansatz that produced a normalizable scalar mode independent of the four-dimensional coordinates $x^{\mu}$ was 13 .

$$
\delta B_{2}=\chi(\tau) d g^{5}, \quad \delta G_{13}=\delta G_{24}=\psi(\tau) .
$$

Our first goal is to find a generalization of this ansatz that will allow us to study the radial excitations of this massless scalar, i.e. the series of modes that exist at non-vanishing $k_{\mu}^{2}=-m_{4}^{2}$. Thus, we must include the dependence of all fields on $x^{\mu}$. Such an ansatz that decouples from other fields at linear order is

$$
\begin{aligned}
\delta F_{3} & =0 \\
\delta F_{5} & =0 \\
\delta B_{2} & =\chi(x, \tau) d g^{5}+\partial_{\mu} \sigma(x, \tau) d x^{\mu} \wedge g^{5} \\
\delta H_{3} \equiv d \delta B_{2} & =\chi^{\prime} d \tau \wedge d g^{5}+\partial_{\mu}(\chi-\sigma) d x^{\mu} \wedge d g^{5}+\partial_{\mu} \sigma^{\prime} d \tau \wedge d x^{\mu} \wedge g^{5}, \\
\delta G_{13}=\delta G_{24} & =\psi(x, \tau) .
\end{aligned}
$$

The ansatz for $\delta B_{2}$ originates from the longitudinal component of a 5-d vector:

$$
\delta B_{2}=\left(A_{\tau} d \tau+A_{\mu} d x^{\mu}\right) \wedge g^{5}
$$

Requiring the 4-d field strength to vanish, $F_{\mu \nu}=0$, restricts $A_{\mu}$ to be of the form $\partial_{\mu}$ acting on a function. Then, choosing

$$
A_{\tau}=-\chi^{\prime}, \quad A_{\mu}=\partial_{\mu}(\sigma-\chi),
$$

we recover the ansatz (2) up to a gauge transformation.

Yet another gauge equivalent way of writing (2) is

$$
\delta B_{2}=(\chi-\sigma) d g^{5}-\sigma^{\prime} d \tau \wedge g^{5} .
$$

The new feature of our ansatz compared to the generalized PT ansatz used in [14, 15] is the presence of the second function in $\delta B_{2}$ which multiplies $d \tau \wedge g^{5}$. Terms of this type, which are allowed by 
the 4-d Lorentz symmetry, turn out to be crucial for studying the modes that are odd under the $\mathcal{I}$-symmetry.

Using $\delta\left(G^{-1}\right)=-G^{-1} \delta G G^{-1}$, we find that $\delta G^{13}=\delta G^{24}=-G^{11} G^{33} \psi$. The unperturbed metric components (see Appendix B.1 for a review of the KS solution) are

$$
\begin{aligned}
G^{11}=G^{22} & =\frac{2}{\epsilon^{4 / 3} K(\tau) \sinh ^{2}(\tau / 2) h^{1 / 2}(\tau)}, \\
G^{33}=G^{44} & =\frac{2}{\epsilon^{4 / 3} K(\tau) \cosh ^{2}(\tau / 2) h^{1 / 2}(\tau)}, \\
G^{55}=G^{\tau \tau} & =\frac{6 K(\tau)^{2}}{\epsilon^{4 / 3} h^{1 / 2}}, \\
G^{\mu \nu} & =h^{1 / 2} \eta^{\mu \nu}
\end{aligned}
$$

In order to find the dynamic equations for the functions $\psi, \chi$ and $\sigma$ in (2) we study the linearized supergravity equations below (type IIB SUGRA equations are reviewed in Appendix (A)).

\subsection{Equations of Motion for NSNS- and RR-Forms}

All the Bianchi identities are automatically satisfied with the ansatz (2). Indeed, the relation $d \delta H_{3}=0$ is obvious, and consistent with vanishing $d F_{5}$ we find that $\delta H_{3} \wedge F_{3}=0$ (using eqs. (141) and (145) one can verify that $d g^{5} \wedge F_{3}=0$ and $d \tau \wedge g^{5} \wedge F_{3}=0$ ).

The self-duality equation for $F_{5}$ reads

$$
\delta * F_{5}=0
$$

Given that $F_{5}$ has components along $g^{1} \wedge g^{2} \wedge g^{3} \wedge g^{4} \wedge g^{5}$ and along $d^{4} x \wedge d \tau$, our adopted deformation of the metric does not affect $* F_{5}$ to first order.

Even though the variations of the forms $F_{3}$ and $F_{5}$ are zero, the deformations of their Hodge duals $\delta * F_{3}$ and $\delta * F_{5}$ will in general be non-zero because of the deformations of metric components. In the equation for $F_{3}$

$$
d \delta * F_{3}=F_{5} \wedge \delta H_{3}
$$

the product $F_{5} \wedge \delta H_{3}$ vanishes identically. From the explicit form of $F_{3}$ we see that the Hodge dual of the first two terms in (141) will be a closed form $\delta * F_{3}=A(x, \tau) d^{4} x \wedge d \tau \wedge(\ldots)$. The third term in (141), $F^{\prime} d \tau \wedge\left(g^{1} \wedge g^{3}+g^{2} \wedge g^{4}\right)$, is not affected by the deformation of the metric, and thus $d \delta * F_{3}=0$ is satisfied identically.

The remaining equations are nontrivial. In particular

$$
d \delta * H_{3}=0,
$$

turns out to be more complicated than the equation for $F_{3}$. The variation

$$
\delta * H_{3}=* \delta H_{3}+\delta_{G} * H_{3}
$$


consists of two parts: $* \delta H_{3}$ accounting for the deformation of the form $H_{3}$ itself, and $\delta_{G} * H_{3}$ arising from the deformation of the Hodge star. Explicit calculation shows that

$$
\begin{aligned}
* \delta H_{3}= & -\sqrt{-G} G^{11} G^{33} G^{55} \chi^{\prime} d^{4} x \wedge d g^{5} \wedge g^{5}-\sqrt{-G} G^{11} G^{33}\left|G^{\mu \mu}\right| \partial_{\mu}(\chi-\sigma) *_{4} d x^{\mu} \wedge d \tau \wedge d g^{5} \wedge g^{5} \\
& +\frac{1}{2} \sqrt{-G}\left(G^{55}\right)^{2}\left|G^{\mu \mu}\right| \partial_{\mu} \sigma^{\prime} *_{4} d x^{\mu} \wedge d g^{5} \wedge d g^{5}, \\
\delta_{G} * H_{3}= & -\frac{g_{s} M \alpha^{\prime}}{2} \sqrt{-G} G^{11} G^{33} G^{55}\left[f^{\prime} G^{11}+k^{\prime} G^{33}\right] \psi d^{4} x \wedge d g^{5} \wedge g^{5} .
\end{aligned}
$$

The four-dimensional Hodge star $*_{4}$ is taken w.r.t. the standard Minkowski metric. Differentiating this expression for $\delta * H_{3}$ and equating to zero the coefficients multiplying linearly independent forms gives three equations:

$$
\begin{aligned}
& d^{4} x \wedge d g^{5} \wedge d g^{5}: \quad 2 G^{11} G^{33}\left[\frac{g_{s} M \alpha^{\prime}}{2}\left[f^{\prime} G^{11}+k^{\prime} G^{33}\right] \psi+\chi^{\prime}\right]=G^{55} h^{\frac{1}{2}} \square_{4} \sigma^{\prime}, \\
& d^{4} x \wedge d \tau \wedge d g^{5} \wedge g^{5}: \quad \partial_{\tau}\left\{\sqrt{-G} G^{11} G^{33} G^{55}\left[\frac{g_{s} M \alpha^{\prime}}{2}\left[f^{\prime} G^{11}+k^{\prime} G^{33}\right] \psi+\chi^{\prime}\right]\right\}+ \\
&+\sqrt{-G} G^{11} G^{33} h^{\frac{1}{2}} \square_{4}(\chi-\sigma)=0 \\
& *_{4} d x^{\mu} \wedge d \tau \wedge d g^{5} \wedge d g^{5}: \quad 2 \sqrt{-G} G^{11} G^{33} h^{\frac{1}{2}} \partial_{\mu}(\chi-\sigma)+\partial_{\tau}\left\{\sqrt{-G}\left(G^{55}\right)^{2} h^{\frac{1}{2}} \partial_{\mu} \sigma^{\prime}\right\}=0
\end{aligned}
$$

where we have substituted for the warp factor $\left|G^{\mu \mu}\right|=h^{\frac{1}{2}}$ (no summation over $\mu$ is implied). Not all of these equations are independent. Indeed, using (15) equation (16) simplifies to

$$
\partial_{\tau}\left\{\sqrt{-G}\left(G^{55}\right)^{2} h^{\frac{1}{2}} \square_{4} \sigma^{\prime}\right\}+2 \sqrt{-G} G^{11} G^{33} h^{\frac{1}{2}} \square_{4}(\chi-\sigma)=0 .
$$

This is exactly what we obtain by acting on (17) with $\partial^{\mu}$ and contracting indices. Thus only (15) and (17) are independent. The coefficient functions in these equations are given by (we have dropped some inessential constant factor in $\sqrt{-G}$ ):

$$
\begin{aligned}
f^{\prime} G^{11}+k^{\prime} G^{33} & =\frac{2(\sinh 2 \tau-2 \tau)}{\epsilon^{4 / 3} \sqrt{h(\tau)} K(\tau) \sinh ^{3} \tau}=\frac{4 K(\tau)^{2}}{\epsilon^{4 / 3} \sqrt{h(\tau)}}, \\
G^{11} G^{33} & =\frac{16}{\epsilon^{8 / 3} h(\tau) K(\tau)^{2} \sinh ^{2} \tau}, \\
\sqrt{h} G^{55} & =\frac{6 K(\tau)^{2}}{\epsilon^{4 / 3}}, \\
\sqrt{-G} G^{11} G^{33} h^{1 / 2} & \sim \frac{4}{K(\tau)^{2}}, \\
\sqrt{-G}\left(G^{55}\right)^{2} h^{1 / 2} & \sim 9 K(\tau)^{4} \sinh ^{2} \tau .
\end{aligned}
$$

Taking into account these expressions, equations (15) and (17) read

$$
\begin{aligned}
2\left(g_{s} M \alpha^{\prime}\right) \frac{K(\tau)^{2}}{\epsilon^{4 / 3} \sqrt{h(\tau)}} \psi+\chi^{\prime} & =\frac{3}{16} \epsilon^{4 / 3} h(\tau) K(\tau)^{4} \sinh ^{2} \tau \square_{4} \sigma^{\prime}, \\
\partial_{\mu}(\chi-\sigma)+\frac{9}{8} K(\tau)^{2} \partial_{\tau}\left\{K^{4} \sinh ^{2} \tau \partial_{\mu} \sigma^{\prime}\right\} & =0 .
\end{aligned}
$$




\subsection{Einstein Equations}

The first order perturbation of the Ricci curvature tensor is given by

$$
\delta R_{i j}=\frac{1}{2}\left(-\delta G_{a}^{a} ; i j-\delta G_{i j ; a}{ }^{a}+\delta G_{a i ; j}{ }^{a}+\delta G_{a j ; i}{ }^{a}\right),
$$

where covariant derivatives and contractions of indices are performed using the unperturbed metric. The first term in this expression vanishes because the metric perturbation is traceless. The remaining three terms combine to give the only non-zero perturbations $\delta R_{13}=\delta R_{24}$ :

$$
\begin{aligned}
\delta R_{13}= & -\frac{3}{\epsilon^{4 / 3}} K^{3} \sinh (\tau) z\left[\frac{K^{\prime \prime}}{K}+\frac{1}{2} \frac{h^{\prime \prime}}{h}+\frac{z^{\prime \prime}}{z}+\frac{\left(K^{\prime}\right)^{2}}{K^{2}}-\frac{1}{2} \frac{\left(h^{\prime}\right)^{2}}{h^{2}}+\frac{K^{\prime}}{K} \frac{h^{\prime}}{h}+\right. \\
& \left.2 \frac{K^{\prime}}{K} \frac{z^{\prime}}{z}+\operatorname{coth} \tau\left(\frac{h^{\prime}}{h}+4 \frac{K^{\prime}}{K}+2 \frac{z^{\prime}}{z}\right)+2-\frac{1}{\sinh (\tau)^{2}}-\frac{4}{9} \frac{1}{\sinh (\tau)^{2} K^{6}}\right]-\frac{1}{2} h(\tau) K \sinh (\tau) \square_{4} z \\
= & -\frac{3}{\epsilon^{4 / 3}} K^{3} \sinh \tau z\left[\frac{1}{2} \frac{\left((K \sinh (\tau))^{2}(\ln h)^{\prime}\right)^{\prime}}{(K \sinh (\tau))^{2}}+\frac{\left((K \sinh (\tau))^{2} z^{\prime}\right)^{\prime}}{(K \sinh (\tau))^{2} z}\right. \\
& \left.-\frac{2}{\sinh (\tau)^{2}}-\frac{8}{9} \frac{1}{K^{6} \sinh (\tau)^{2}}+\frac{4}{3} \frac{\cosh (\tau)}{K^{3} \sinh (\tau)^{2}}\right]-\frac{1}{2} h(\tau) K \sinh \tau \square_{4} z,
\end{aligned}
$$

where $z(x, \tau)$ is defined by

$$
\psi(x, \tau)=h^{1 / 2} K \sinh (\tau) z(x, \tau)=2^{-1 / 3}[\sinh (2 \tau)-2 \tau]^{1 / 3} h^{1 / 2} z(x, \tau) .
$$

The source terms on the right hand side of the Einstein equation $R_{i j}=T_{i j}$ (120) are due to the deformations of the metric and $B_{2}$ form. It turns out that the only nontrivial deformations are those with indices 13 or 24 , with $\delta T_{13}=\delta T_{24}$. Say, for the 13 component $\delta T_{13}$ we have the following contributions:

$$
\begin{aligned}
\frac{1}{4} \delta_{B}\left(H_{1 a b} H_{3}{ }^{a b}\right) & =\frac{1}{4}\left[H_{1 a b} \delta H_{3}{ }^{a b}+\delta H_{1 a b} H_{3}{ }^{a b}\right] \\
& =\frac{1}{2}\left[G^{11} H_{12 \tau} \delta H_{32 \tau}+G^{33} \delta H_{14 \tau} H_{34 \tau}\right] G^{55} \\
& =-\frac{1}{4}\left(g_{s} M \alpha^{\prime}\right) G^{55}\left[G^{11} f^{\prime}+G^{33} k^{\prime}\right] \chi^{\prime}, \\
\frac{g_{s}^{2}}{96} \delta_{G}\left(F_{1 a b c d} F_{3}{ }^{a b c d}\right) & =\frac{g_{s}^{2}}{4}\left(G^{11}\right)^{2}\left(G^{33}\right)^{2} G^{55}\left(F_{12345}\right)^{2} \psi \\
\frac{1}{4} \delta_{G}\left(H_{1 a b} H_{3}{ }^{a b}\right) & =\frac{1}{2}\left[H_{135} H_{315} \delta G^{13} G^{55}+H_{12 \tau} H_{34 \tau} \delta G^{24} G^{\tau \tau}\right] \\
& =\frac{1}{2}\left[\left(H_{135}\right)^{2}-H_{12 \tau} H_{34 \tau}\right] G^{11} G^{33} G^{55} \psi \\
& =\frac{1}{8}\left(g_{s} M \alpha^{\prime}\right)^{2}\left[\frac{1}{4}(k-f)^{2}-f^{\prime} k^{\prime}\right] G^{11} G^{33} G^{55} \psi,
\end{aligned}
$$




$$
\begin{aligned}
\frac{g_{s}^{2}}{4} \delta_{G}\left(F_{1 a b} F_{3}{ }^{a b}\right)= & \frac{g_{s}^{2}}{2}\left[F_{125} F_{345} \delta G^{24} G^{55}+F_{13 \tau} F_{31 \tau} \delta G^{31} G^{\tau \tau}\right] \\
= & \frac{g_{s}^{2}}{2}\left[\left(F_{13 \tau}\right)^{2}-F_{125} F_{345}\right] G^{11} G^{33} G^{55} \psi \\
= & \frac{1}{8}\left(g_{s} M \alpha^{\prime}\right)^{2}\left[F^{\prime 2}-F(1-F)\right] G^{11} G^{33} G^{55} \psi \\
-\frac{1}{48} \delta_{G}\left[G_{13}\left(H_{a b c} H^{a b c}+g_{s}^{2} F_{a b c} F^{a b c}\right)\right]= & -\frac{1}{8}\left(H^{2}+g_{s}^{2} F^{2}\right) \psi \\
= & -\frac{1}{32}\left(g_{s} M \alpha^{\prime}\right)^{2} G^{55}\left[\left(G^{11}\right)^{2} f^{\prime 2}+\left(G^{33}\right)^{2} k^{\prime 2}\right. \\
& +\frac{1}{2} G^{11} G^{33}(k-f)^{2}+\left(G^{11}\right)^{2} F^{2}+\left(G^{33}\right)^{2}(1-F)^{2} \\
& \left.+2 G^{11} G^{33} F^{\prime 2}\right] \psi
\end{aligned}
$$

Denoting

$$
\delta T_{13}=\left[A_{1}(\tau)+A_{2}(\tau)\right] \psi(x, \tau)+B(\tau) \chi^{\prime}(x, \tau),
$$

where $A_{1}$ stands for the contribution from $F_{5}$, we get

$$
\begin{aligned}
& A_{1}(\tau)=\frac{3\left(g_{s} M \alpha^{\prime}\right)^{4}}{2^{1 / 3} \epsilon^{20 / 3} h^{5 / 2}} \frac{(\tau \operatorname{coth} \tau-1)^{2}[\sinh (2 \tau)-2 \tau]^{4 / 3}}{\sinh ^{6}(\tau)} \\
& A_{2}(\tau)=-\frac{3\left(g_{s} M \alpha^{\prime}\right)^{2}}{8 \epsilon^{4} h^{3 / 2} \sinh ^{6} \tau}\left[3 \cosh 4 \tau-8 \tau \sinh 2 \tau-8 \tau^{2} \cosh 2 \tau-8 \cosh 2 \tau+16 \tau^{2}+5\right], \\
& B(\tau)=-3\left(g_{s} M \alpha^{\prime}\right) \frac{(\sinh 2 \tau-2 \tau) K(\tau)}{\epsilon^{8 / 3} h(\tau) \sinh ^{3} \tau} .
\end{aligned}
$$

Then eliminating $\chi^{\prime}$ with the help of (15) yields

$$
\begin{aligned}
\delta T_{13}= & \frac{3}{2^{2 / 3}} \frac{\left(g_{s} M \alpha^{\prime}\right)^{4}}{\epsilon^{20 / 3} h^{2}} \frac{(\tau \operatorname{coth} \tau-1)^{2}[\sinh 2 \tau-2 \tau]^{5 / 3}}{\sinh ^{6} \tau} z(\tau)+\frac{3}{8 \cdot 2^{1 / 3}} \frac{\left(g_{s} M \alpha^{\prime}\right)^{2}}{\epsilon^{4} h} \frac{(\sinh (2 \tau)-2 \tau)^{1 / 3}}{\sinh ^{6}(\tau)} \times \\
& \times\left[\cosh (4 \tau)+8\left(1+\tau^{2}\right) \cosh (2 \tau)-24 \tau \sinh (2 \tau)+16 \tau^{2}-9\right] z(x, \tau) \\
& -\frac{9}{16} \frac{g_{s} M \alpha^{\prime}}{\epsilon^{4 / 3}} \frac{\sinh 2 \tau-2 \tau}{\sinh \tau} K^{5} \square_{4} \sigma^{\prime}(x, \tau) \\
= & -\frac{3}{\epsilon^{4 / 3}} K^{3} \sinh \tau\left[-\frac{1}{2} \frac{\left(h^{\prime}\right)^{2}}{h^{2}}+\frac{1}{2} \frac{h^{\prime \prime}}{h}+\frac{K^{\prime}}{K} \frac{h^{\prime}}{h}+\operatorname{coth} \tau \frac{h^{\prime}}{h}\right] z-\frac{9}{16} \frac{g_{s} M \alpha^{\prime}}{\epsilon^{4 / 3}} \frac{\sinh 2 \tau-2 \tau}{\sinh \tau} K^{5} \square_{4} \sigma^{\prime} .
\end{aligned}
$$

As mentioned above the perturbations $\delta T_{13}=\delta T_{24}$ are the only non-zero components of $\delta T_{i j}$. Equating (27) and (38) we obtain the final form of the linearized Einstein equation. 


\subsection{Two Coupled Scalars}

Combining the equations for the field strengths and the Einstein equations we have the system

$$
\begin{aligned}
\left(g_{s} M \alpha^{\prime}\right) \frac{\sinh 2 \tau-2 \tau}{\epsilon^{4 / 3} \sinh ^{2} \tau} z+\chi^{\prime}= & \frac{3}{16} \epsilon^{4 / 3} h(\tau) K(\tau)^{4} \sinh ^{2} \tau \square_{4} \sigma^{\prime}, \\
\partial_{\mu}(\chi-\sigma)= & -\frac{9}{8} K(\tau)^{2} \partial_{\tau}\left\{K^{4} \sinh ^{2} \tau \partial_{\mu} \sigma^{\prime}\right\}, \\
\frac{\left((K \sinh \tau)^{2} z^{\prime}\right)^{\prime}}{(K \sinh \tau)^{2}}+\frac{\epsilon^{4 / 3} h}{6 K^{2}} \square_{4} z= & \left(\frac{2}{\sinh ^{2} \tau}+\frac{8}{9} \frac{1}{K^{6} \sinh ^{2} \tau}-\frac{4}{3} \frac{\cosh \tau}{K^{3} \sinh ^{2} \tau}\right) z \\
& +\frac{3}{16}\left(g_{s} M \alpha^{\prime}\right) \frac{\sinh ^{2} \tau-2 \tau}{\sinh ^{2} \tau} K^{2} \square_{4} \sigma^{\prime} .
\end{aligned}
$$

Note that $\chi$ can be eliminated between (39) and (40). Further, a change of variables

$$
\begin{aligned}
\tilde{z} & =z K \sinh (\tau), \\
\tilde{w} & =\frac{\epsilon^{4 / 3}}{g_{s} M \alpha^{\prime}} K^{5} \sinh (\tau)^{2} \sigma^{\prime},
\end{aligned}
$$

leads to a more symmetric pair of equations

$$
\begin{aligned}
\tilde{z}^{\prime \prime}-\frac{2}{\sinh ^{2} \tau} \tilde{z}+\frac{\epsilon^{4 / 3} h}{6 K^{2}} \square_{4} \tilde{z}=\frac{3\left(g_{s} M \alpha^{\prime}\right)^{2}}{16 \epsilon^{4 / 3}} \frac{\sinh 2 \tau-2 \tau}{K^{2} \sinh ^{3} \tau} \square_{4} \tilde{w}, \\
\tilde{w}^{\prime \prime}-\frac{\cosh ^{2} \tau+1}{\sinh ^{2} \tau} \tilde{w}+\frac{\epsilon^{4 / 3} h}{6 K^{2}} \square_{4} \tilde{w}=\frac{8}{9} \frac{\sinh 2 \tau-2 \tau}{K^{2} \sinh ^{3} \tau} \tilde{z} .
\end{aligned}
$$

Introducing the dimensionless mass-squared $\tilde{m}^{2}$ according to

$$
\tilde{m}^{2}=m_{4}^{2} \frac{2^{2 / 3}\left(g_{s} M \alpha^{\prime}\right)^{2}}{6 \epsilon^{4 / 3}},
$$

we can rewrite the equations for $\tilde{z}$ and $\tilde{w}$ as

$$
\begin{aligned}
\tilde{z}^{\prime \prime}-\frac{2}{\sinh ^{2} \tau} \tilde{z}+\tilde{m}^{2} \frac{I(\tau)}{K^{2}(\tau)} \tilde{z} & =\tilde{m}^{2} \frac{9}{4 \cdot 2^{2 / 3}} K(\tau) \tilde{w}, \\
\tilde{w}^{\prime \prime}-\frac{\cosh ^{2} \tau+1}{\sinh ^{2} \tau} \tilde{w}+\tilde{m}^{2} \frac{I(\tau)}{K^{2}(\tau)} \tilde{w} & =\frac{16}{9} K(\tau) \tilde{z} .
\end{aligned}
$$

This is a system of coupled equations which defines the mass spectrum of certain scalar glueballs with positive 4-d parity. The natural charge conjugation symmetry of the KS background is the $\mathcal{I}$-symmetry, under which these modes are odd. Therefore, we assign $J^{P C}=0^{+-}$to this family of glueballs 1

In the massless case these equations lead to the GHK solution [13. If we assume $\square_{4}=-k_{\mu}^{2}=$ $m_{4}^{2}=0$, then there are two solutions [13], $\tilde{z}_{1}=\operatorname{coth} \tau$ and $\tilde{z}_{2}=\tau \operatorname{coth} \tau-1$. The solution for $\tilde{z}$

\footnotetext{
${ }^{1}$ For comparison, the glueballs found in [14, 15] are $0^{++}$. The glueballs whose spectrum comes from the minimal scalar equation [1] resulting from the analysis of graviton fluctuations are $2^{++}$. The axial vector $U(1)_{R}$ fluctuations [17 give rise to $1^{++}$glueballs whose masses are also determined by the minimal scalar equation.
} 
which is non-singular at the origin is $\tilde{z}=\tau \operatorname{coth} \tau-1$. Substituting it into the second equation, we find

$$
\tilde{w}^{\prime \prime}-\frac{\cosh ^{2} \tau+1}{\sinh ^{2} \tau} \tilde{w}=\frac{16}{9} K(\tau)(\tau \operatorname{coth} \tau-1) \equiv-\frac{2^{2 / 3} 8}{9} I^{\prime}(\tau) \sinh \tau .
$$

The two solutions of the homogeneous equation are $\tilde{w}_{1}=1 / \sinh \tau$ and $\tilde{w}_{2}=(\sinh 2 \tau-2 \tau) / \sinh \tau$; both of them are singular either at zero or at infinity. This means that the regular solution of the inhomogeneous equation is uniquely fixed. With the Wronskian $W\left(\tilde{w}_{1}, \tilde{w}_{2}\right)=\tilde{w}_{1} \tilde{w}_{2}^{\prime}-\tilde{w}_{1}^{\prime} \tilde{w}_{2}=4$, we can find a general solution

$$
\tilde{w}(\tau)=-\frac{2^{2 / 3} 8}{9}\left\{\tilde{w}_{1}(\tau)\left[C_{1}-\int^{\tau} d x \frac{\tilde{w}_{2}(x)}{W(x)} I^{\prime}(x) \sinh x\right]+\tilde{w}_{2}(\tau)\left[C_{2}+\int^{\tau} d x \frac{\tilde{w}_{1}(x)}{W(x)} I^{\prime}(x) \sinh x\right]\right\} .
$$

Integrating by parts and choosing the particular homogeneous solution to make $\tilde{w}$ well behaved at both zero and infinity we get

$$
\tilde{w}(\tau)=-\frac{2^{2 / 3} 8}{9} \frac{1}{\sinh \tau} \int_{0}^{\tau} d x I(x) \sinh ^{2} x .
$$

Alternatively, if we start with equation (3.18) in the GHK paper [13], which determines $f_{2}$, and introduce $\tilde{w}=f_{2}(\tau) K^{2} \sinh \tau$, we get (49), up to a rescaling of the right hand side. Thus, we can use the solution (3.25) of [13] for $f_{2}(\tau)$ to read off the result (51).

Let us also note that the non-zero $\tilde{w}$ in the zero momentum case $k_{\mu}=0$ is not in contradiction with the GHK solution. This is because $\tilde{w}$ enters (2) only through $\partial_{\mu} \sigma$ which is zero as long as the momentum vanishes.

\subsection{Numerical Analysis}

To determine the spectrum of glueballs in the field theory, we need to solve the eigenvalue problem for $\tilde{m}^{2}$ in the infinite throat limit. This system of equations (47), (48) does not seem amenable to analytical solution and we employ a numerical approach to find the spectrum of normalizable solutions. It is convenient to use the determinant method, which generalizes the standard shooting technique to a system of several coupled equations (see [15]). The detailed description of the numerical analysis as well as of the subtleties specific to the system (47), (48) is given in Appendix D. The result is that the spectrum consists of two distinct series, each with a quadratic growth of $\tilde{m}_{n}^{2}$ for large $n$. These series are interpreted as the radial excitation spectra of two different particles. The lowest eigenvalues $\left(\tilde{m}^{2}<100\right)$ for these spectra are shown in Table 1. The quadratic fit for spectrum I is

$$
\tilde{m}_{I n}^{2}=2.31+1.91 n+0.294 n^{2} .
$$

For spectrum II (we drop the lowest eigenvalue when doing the fit)

$$
\tilde{m}_{I I n}^{2}=0.36+0.14 n+0.279 n^{2} .
$$


Table 1: Non-zero eigenvalues with $\tilde{m}^{2}<100$. There are the two distinct spectra. Both spectra can be fitted by quadratic polynomials in the eigenvalue number $n$ (the red line in the plots).

Spectrum I

\begin{tabular}{||c|c||c|c||c|c||c|c||}
\hline$n$ & $\tilde{m}_{n}^{2}$ & $n$ & $\tilde{m}_{n}^{2}$ & $n$ & $\tilde{m}_{n}^{2}$ & $n$ & $\tilde{m}_{n}^{2}$ \\
\hline 1 & 4.53 & 5 & 19.1 & 9 & 43.3 & 13 & 76.9 \\
\hline 2 & 7.30 & 6 & 24.4 & 10 & 50.8 & 14 & 86.7 \\
\hline 3 & 10.7 & 7 & 30.1 & 11 & 58.9 & 15 & 97.1 \\
\hline 4 & 14.6 & 8 & 36.4 & 12 & 67.6 & & \\
\hline
\end{tabular}

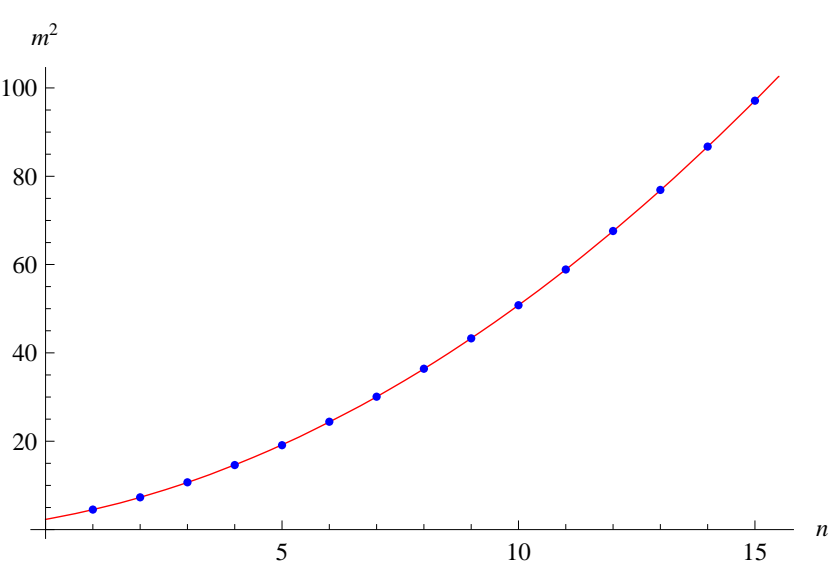

Spectrum II

\begin{tabular}{||c|c||c|c||c|c||c|c||}
\hline$n$ & $\tilde{m}_{n}^{2}$ & $n$ & $\tilde{m}_{n}^{2}$ & $n$ & $\tilde{m}_{n}^{2}$ & $n$ & $\tilde{m}_{n}^{2}$ \\
\hline 1 & 0.129 & 6 & 8.06 & 11 & 30.1 & 16 & 65.1 \\
\hline 2 & 0.703 & 7 & 11.2 & 12 & 35.5 & 17 & 73.9 \\
\hline 3 & 1.76 & 8 & 15.0 & 13 & 42.1 & 18 & 83.3 \\
\hline 4 & 3.33 & 9 & 19.3 & 14 & 49.2 & 19 & 93.3 \\
\hline 5 & 5.43 & 10 & 24.1 & 15 & 56.9 & & \\
\hline
\end{tabular}

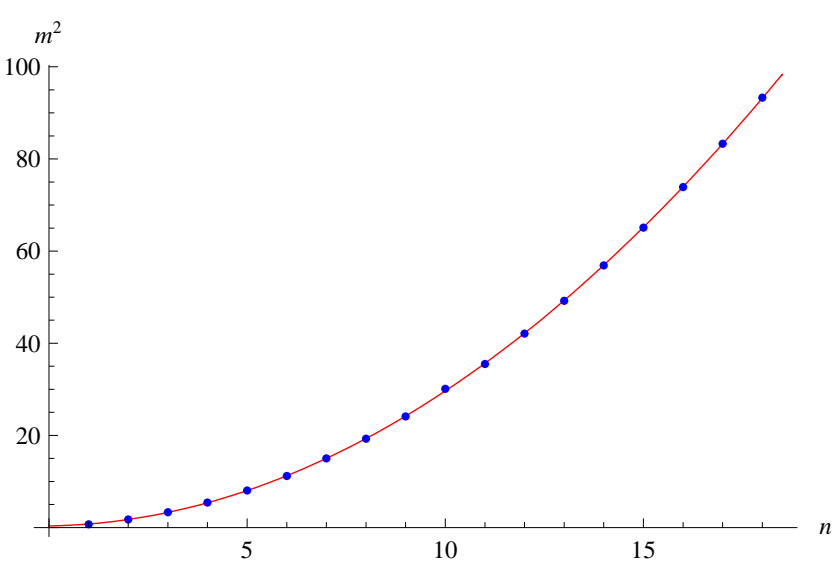

It is interesting to compare these results with those found for the $0^{++}$modes by Berg, Haack and Mück (BHM) [15]. The conventions of [15] correspond to a particular choice of the KS parameters (see Appendix (B.3), and the relation between the masses is

$$
m_{B H M}^{2}=(3 / 2)^{2 / 3} I(0) \tilde{m}^{2} \approx 0.9409 \tilde{m}^{2} .
$$

Using this relation one can convert the mass eigenvalues to the BHM normalization. We note that the lightest glueball we find, the first entry from spectrum II in Table 1 , has $m_{B H M}^{2} \approx 0.121$. For comparison, the lightest $0^{++}$eigenvalue found in [15] has $m_{B H M}^{2} \approx 0.185$. The fact that the $0^{+-}$sector has the lightest glueballs may be qualitatively understood as follows. Roughly speaking, glueball masses increase with the dimensions of the operators that create them. The lowest dimension operator from the $0^{++}$sector is the gluino bilinear $\operatorname{Tr} \lambda \lambda$ of dimension 3 , but the $0^{+-}$sector contains an operator of dimension 2, namely $\operatorname{Tr}(\bar{A} A-\bar{B} B)$.

Converting the asymptotics of the two spectra to BHM units, we find

$$
\begin{aligned}
m_{I B H M}^{2} & \approx 2.17+1.79 n+0.277 n^{2}, \\
m_{I I_{B H M}}^{2} & \approx 0.34+0.13 n+0.262 n^{2} .
\end{aligned}
$$


The coefficients of the quadratic terms are close to those found in [15]. The quadratic dependence on $n$, which is characteristic of Kaluza-Klein theory, is a special feature of strongly coupled gauge theories that have weakly curved gravity duals (see [29] for a discussion). Note that $m_{4}^{2}$ is obtained from $\tilde{m}^{2}$ through multiplying by a factor $\sim T_{s} /\left(g_{s} M\right)$, where $T_{s}$ is the confining string tension. Thus, for $n \ll \sqrt{g_{s} M}$ these modes are much lighter than the string tension scale, and therefore much lighter than all glueballs with spin $>2$. Such anomalously light bound states appear to be

special to gauge theories that stay very strongly coupled in the UV, such as the cascading gauge theory; they do not appear in asymptotically free gauge theories. Therefore, the anomalously light glueballs could perhaps be used as a 'special signature' of gauge theories with gravity duals if they are realized in nature.

One may be puzzled why the spectrum in Table1 does not include the GHK massless mode. This is because in solving the coupled equations (47), (48) we required that both wave-functions $\tilde{z}$ and $\tilde{w}$ vanish as $\tau \rightarrow \infty$. This excludes the GHK zero mode which grows as $\tilde{z} \sim \tau$. On the other hand, this growth is a lot slower than the exponential growth found for generic solutions. The meaning of the GHK mode as the baryonic branch modulus seems to be well established since even the solutions at finite distance along this modulus are available [20, 21]. Thus, the GHK scalar zero-mode should be normalizable with a proper definition of norm. In fact, the GHK pseudoscalar and its fermionic superpartner are normalizable [13, 16]; therefore, the supersymmetry of the problem implies that the GHK scalar is normalizable as well and is part of the spectrum.

\section{Pseudoscalar Modes from the RR Sector}

The type of ansatz used in section 2 works even more simply for the RR 2-form field:

$$
\begin{aligned}
\delta H_{3} & =0 \\
\delta F_{5} & =0 \\
\delta C_{2} & =\chi(x, \tau) d g^{5}+\partial_{\mu} \sigma(x, \tau) d x^{\mu} \wedge g^{5} \\
\delta F_{3} \equiv d \delta C_{2} & =\chi^{\prime} d \tau \wedge d g^{5}+\partial_{\mu}(\chi-\sigma) d x^{\mu} \wedge d g^{5}+\partial_{\mu} \sigma^{\prime} d \tau \wedge d x^{\mu} \wedge g^{5} .
\end{aligned}
$$

This ansatz is similar to, but somewhat simpler than the GHK pseudoscalar ansatz [13] which involved mixing with $\delta F_{5}$. Since $\delta F_{3} \wedge H_{3}=0$, now it is consistent to set $\delta F_{5}=0$. We also have $F_{5} \wedge \delta F_{3}=0$, so it is consistent to take $\delta H_{3}=0$. Finally, one needs to study mixing with metric fluctuations. At a first glance it seems that $\delta G_{12}$ and $\delta G_{34}$ might need to be turned on, but a more detailed analysis shows that their sources vanish:

$$
\begin{aligned}
& \delta T_{12}=F_{13 \tau} \delta F_{2}^{3 \tau}+\delta F_{14 \tau} F_{2}{ }^{4 \tau}=\frac{M \alpha^{\prime}}{2} G^{33} G^{55}\left[F^{\prime} \chi^{\prime}-F^{\prime} \chi^{\prime}\right]=0, \\
& \delta T_{34}=F_{31 \tau} \delta F_{4}{ }^{1 \tau}+\delta F_{32 \tau} F_{4}{ }^{2 \tau}=0 .
\end{aligned}
$$


Thus, the perturbation (57) decouples from all other modes, and the only non-trivial linearized equation is

$$
d * \delta F_{3}=0
$$

The calculation we need to perform is the same as in section 2.1 , except we now set $\psi=0$ and find

$$
\begin{gathered}
\chi^{\prime}=\frac{3}{16} \epsilon^{4 / 3} h(\tau) K(\tau)^{4} \sinh ^{2} \tau \square_{4} \sigma^{\prime}, \\
\partial_{\mu}(\chi-\sigma)+\frac{9}{8} K(\tau)^{2} \partial_{\tau}\left\{K^{4} \sinh ^{2} \tau \partial_{\mu} \sigma^{\prime}\right\}=0 .
\end{gathered}
$$

Eliminating $\chi$ and changing variables as before,

$$
\tilde{w}=\frac{\epsilon^{4 / 3}}{g_{s} M \alpha^{\prime}} K^{5} \sinh (\tau)^{2} \sigma^{\prime}
$$

we find

$$
\tilde{w}^{\prime \prime}-\frac{\cosh ^{2} \tau+1}{\sinh ^{2} \tau} \tilde{w}+\frac{\epsilon^{4 / 3} h}{6 K^{2}} \square_{4} \tilde{w}=0 .
$$

Again, after introducing the dimensionless mass as in (46), we get a non-minimal scalar equation

$$
\tilde{w}^{\prime \prime}-\frac{\cosh ^{2} \tau+1}{\sinh ^{2} \tau} \tilde{w}+\tilde{m}^{2} \frac{I(\tau)}{K(\tau)^{2}} \tilde{w}=0 .
$$

Since the 4-d parity operation includes sign reversal of RR fields, we identify the family of glueballs coming from this eigenvalue problem as pseudoscalars whose $J^{P C}$ quantum numbers are $0^{--}$.

If we set $\tilde{m}=0$ the solution regular at small $\tau$ is $(\sinh 2 \tau-2 \tau) / \sinh \tau$. Since this blows up at large $\tau$ we conclude that this equation does not contain a massless glueball. A simple numerical analysis using the shooting method allows one to find the mass spectrum. The lowest eigenvalues $\left(\tilde{m}^{2}<100\right)$ are listed in Table 2. The quadratic fit is

$$
\tilde{m}_{I I I n}^{2}=0.996+1.15 n+0.289 n^{2}
$$

in the BHM normalization it is given by

$$
m_{I I I \text { BHM }}^{2}=0.938+1.08 n+0.272 n^{2} .
$$

The spectrum can be reproduced with good accuracy using a semiclassical (WKB) approximation. The effective potential in (65) is singular at $\tau=0$ which does not allow us to use the conventional WKB approximation. Yet we can cast the equation (65) in the form $Q_{1} Q_{2} \tilde{w}=m^{2} \tilde{w}$, where $Q_{i}$ are first-order differential operators and then consider an equation $Q_{2} Q_{1} \tilde{\tilde{w}}=m^{2} \tilde{\tilde{w}}$, which must give rise to the same spectrum up to a zero mode. Namely, in our case this means that for $A$ such that

$$
A^{2}+A^{\prime}=\frac{\cosh ^{2} \tau+1}{\sinh ^{2} \tau}
$$


Table 2: Non-zero eigenvalues with $\tilde{m}^{2}<100$ in the RR sector. This spectrum can also be fitted by a quadratic polynomial (red line).

\begin{tabular}{||c|c||c|c||c|c||c|c||}
\hline$n$ & $\tilde{m}_{n}^{2}$ & $n$ & $\tilde{m}_{n}^{2}$ & $n$ & $\tilde{m}_{n}^{2}$ & $n$ & $\tilde{m}_{n}^{2}$ \\
\hline 1 & 2.41 & 5 & 14.0 & 9 & 34.8 & 13 & 64.7 \\
\hline 2 & 4.47 & 6 & 18.0 & 10 & 41.4 & 14 & 73.7 \\
\hline 3 & 7.08 & 7 & 23.2 & 11 & 48.6 & 15 & 83.2 \\
\hline 4 & 10.3 & 8 & 28.7 & 12 & 56.4 & 16 & 93.3 \\
\hline
\end{tabular}

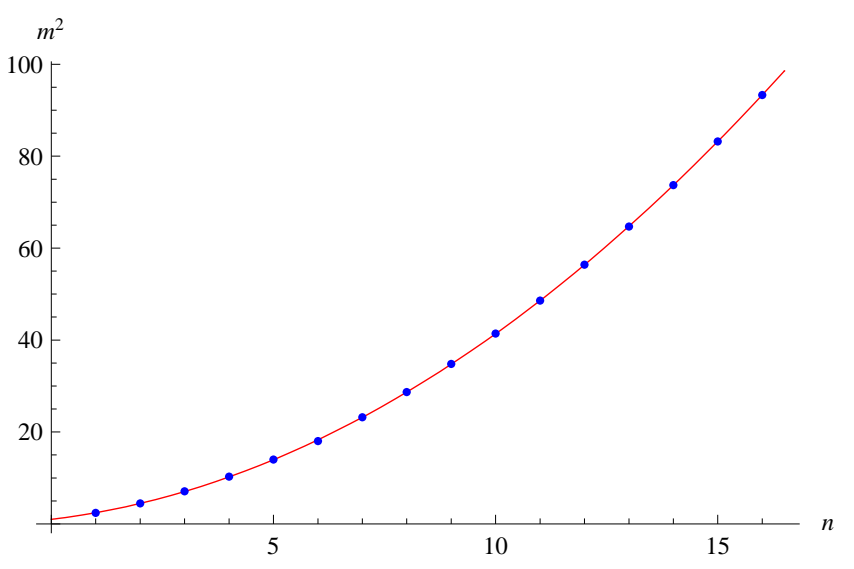

equation (65) shares the spectrum with an equation

$$
\begin{array}{r}
\tilde{\tilde{w}}^{\prime \prime}-\left(B^{2}+B^{\prime}\right) \tilde{\tilde{w}}+\tilde{m}^{2} \frac{I(\tau)}{K(\tau)^{2}} \tilde{\tilde{w}}=0, \\
B=-A-\frac{1}{2} \frac{d}{d \tau} \log \frac{I(\tau)}{K(\tau)^{2}} .
\end{array}
$$

A general solution of (68) reads

$$
A=-\operatorname{coth} \tau+\frac{2 \sinh ^{2} \tau}{\cosh \tau \sinh \tau-\tau+\mathbf{C}}
$$

For (69) to be non-singular at the origin $\mathbf{C}$ has to be non-zero. For a finite $\mathbf{C}$ the potential is regular everywhere but not monotonic and (69) admits a zero mode. A most convenient choice is to take infinite $\mathbf{C}$, which reduces $A$ to $A=-\operatorname{coth} \tau$. In this case the WKB approximation is applicable in it simplest form (see [11] for similar considerations) and yields the same result as the shooting method up to the third digit.

\section{Organizing the Modes into Supermultiplets}

The pseudoscalar Goldstone mode and the massless scalar found in [13] belong to a 4-dimensional chiral multiplet. These fields appear as the phase and the modulus of the baryonic order parameters that vary along the baryonic branch. When a long KS throat is embedded into a Calabi-Yau compactification with fluxes, the baryonic $U(1)$ symmetry becomes gauged and a supersymmetric version of the Higgs mechanism is expected to take place. The axial vector $U(1)_{B}$ gauge field 'eats' the pseudoscalar mode and acquires a mass degenerate with the mass of a scalar Higgs. These fields constitute the bosonic content of a massive $\mathcal{N}=1$ axial vector supermultiplet. 
In the present paper we explicitly constructed the massive modes that are radial excitations of the GHK scalar. It is, of course, interesting to find the supermultiplets they belong to. We will argue that each of these scalar radial excitations is also a member of a massive axial vector supermultiplet. Similarly, each pseudoscalar glueball found in section 3 is a member of a massive vector multiplet. To prove these facts we would need to demonstrate the existence of the $J^{P C}=1^{+-}$glueballs degenerate with the $0^{+-}$glueballs found in section 2, as well as of $1^{--}$glueballs degenerate with the $0^{--}$glueballs found in section 3 . Unfortunately, constructing decoupled equations for vector supergravity fluctuations around the KS background is a difficult task. Instead, we will provide some evidence for our claims by studying axial vector and vector fluctuation equations in the large radius (KT) limit (setting $\alpha^{\prime}=g_{s}=1, N=0$ and $M=2$; see Appendix B.2).

First we reconsider the simple decoupled pseudoscalar equation from the RR sector (64) and argue that its superpartner is given by the four-dimensional vector $A_{1}$ in

$$
\delta B_{2}=A_{1} \wedge g^{5}
$$

where we have chosen the ansatz so that the corresponding radial component $\sim d r \wedge g^{5}$ vanishes. The equation for $d * H_{3}$ implies (with primes denoting derivatives with respect to $r$ )

$$
\left[\frac{r^{3}}{6} *_{4} A_{1}^{\prime}\right]^{\prime}-\frac{4 r}{3} *_{4} A_{1}-\frac{h r^{3}}{6} d_{4} *_{4} d_{4} A_{1}=0
$$

and $d_{4} *_{4} A_{1}=0$, i.e. the vector is divergence-free. Since the Laplacian acting on such a vector is $\square_{4}=-*_{4} d_{4} *_{4} d_{4}$ (note the Minkowski signature of the four dimensional metric), we find

$$
\left[\frac{r^{3}}{6} A_{1}^{\prime}\right]^{\prime}-\frac{4 r}{3} A_{1}+\frac{h r^{3}}{6} \square_{4} A_{1}=0
$$

Defining a new variable $\tilde{A}_{1}=r A_{1}$, it is easy to see that its equation of motion,

$$
\frac{r}{3}\left[\frac{r}{3} \tilde{A}_{1}^{\prime}\right]^{\prime}-\tilde{A}_{1}+\frac{h r^{2}}{9} \square_{4} \tilde{A}_{1}=0
$$

coincides with the KT-limit of the equation for the decoupled pseudoscalar $\tilde{w}$, once we identify $r \sim \epsilon^{2 / 3} e^{\tau / 3}$. In fact, if we make the same ansatz (72) in the full KS background, the equation of motion for $\tilde{A}_{1}=K^{2} \sinh \tau A_{1}$ resulting from the terms $\sim d^{3} x \wedge d \tau \wedge \omega_{2} \wedge \omega_{2}$ in $d * H_{3}=0$ is precisely as in (64):

$$
\frac{d^{2}}{d \tau^{2}} \tilde{A}_{1}-\frac{\cosh ^{2} \tau+1}{\sinh ^{2} \tau} \tilde{A}_{1}+\frac{\epsilon^{4 / 3} h}{6 K^{2}} \square_{4} \tilde{A}_{1}=0
$$

However, this ansatz is not closed in the KS case. The Bianchi identity for $F_{5}$ is not satisfied, so this NSNS vector must mix with RR excitations of $F_{3}$ and/or $F_{5}$ in the KS background. It would be interesting to solve this mixing problem.

Let us now turn to the massive axial vector superpartners of the coupled scalars (44), (45) found above. We make the following ansatz, which is similar to the one studied in [30],

$$
\begin{aligned}
& \delta C_{4}=B_{1} \wedge \omega_{3}+F_{2} \wedge \omega_{2}+K_{1} \wedge d r \wedge \omega_{2}, \\
& \delta C_{2}=C_{1} \wedge g^{5}+D_{2}+E_{1} \wedge d r \\
& \delta B_{2}=H_{2}+J_{1} \wedge d r
\end{aligned}
$$


where $B_{1}, C_{1}, E_{1}, J_{1}, K_{1}$ are axial vectors and $D_{2}, F_{2}, H_{2}$ are two-forms in four dimensions. We choose to split the six degrees of freedom residing in the two-form into a vector and a dual vector, e.g. $D_{2}=d_{4}(\ldots)+*_{4} d_{4} D_{1}$. The degrees of freedom contained in the former (exact) part are in fact the same as those in $E_{1}$, so we can simply write $D_{2}=*_{4} d_{4} D_{1}$ without loss of generality. Similarly, $F_{2}=*_{4} d_{4} F_{1}$ and $H_{2}=*_{4} d_{4} H_{1}$, and the corresponding exact parts can be absorbed into the vectors $K_{1}$ and $J_{1}$, respectively.

The equations of motion then imply that $B_{1}$ and $C_{1}$ have to be divergence-free: $d_{4} *_{4} B_{1}=$ $d_{4} *_{4} C_{1}=0$. If this were not the case their divergences would simply couple to additional scalars $\delta C_{4} \sim d r \wedge \omega_{3}$ and $\delta C_{2} \sim d r \wedge g^{5}$, respectively, but we will not consider this here (i.e. as for $A_{1}$ above we choose as gauge in which these radial components vanish). In fact we will assume that all vectors in our ansatz are divergence-free, and that the terms appearing in the RR- and NSNS-potential.2 are eigenstates of the Laplacian $\square_{4}=-*_{4} d_{4} *_{4} d_{4}$ with eigenvalue $m^{2}$.

We have relegated the details of the derivation of the equations of motion to Appendix C. Splitting the equations obtained from (119) into exact and coexact parts w.r.t. the four-dimensional derivative operator $d_{4}$ shows that the vectors $E_{1}, H_{1}$ and $K_{1}$ decouple 3 . The resulting equations for the remaining vectors read

$$
\begin{gathered}
{\left[\frac{3}{h r} B_{1}^{\prime}\right]^{\prime}+\frac{3}{r} \square_{4} B_{1}=-\frac{3}{r} \square_{4} D_{1},} \\
{\left[\frac{r}{3} F_{1}^{\prime}\right]^{\prime}+\frac{h r}{3} \square_{4} F_{1}=J_{1}+\frac{3}{r} C_{1},} \\
{\left[\frac{r^{3}}{6} C_{1}^{\prime}\right]^{\prime}-\frac{4 r}{3} C_{1}+\frac{h r^{3}}{6} \square_{4} C_{1}=\frac{3}{r} \square_{4} F_{1}-\frac{9}{h r^{2}} B_{1}^{\prime},} \\
{\left[\frac{h r^{5}}{54} D_{1}^{\prime}\right]^{\prime}+\frac{h^{2} r^{5}}{54} \square_{4} D_{1}=-F_{1}^{\prime}-\frac{3}{r} B_{1}-3 \log \frac{r}{r_{*}} J_{1},} \\
{\left[\frac{h r^{5}}{54} J_{1}\right]^{\prime}+3 \log \frac{r}{r_{*}} D_{1}^{\prime}=F_{1}^{\prime}+\frac{3}{r} B_{1},} \\
\square F_{1}-\frac{3}{h r} B_{1}^{\prime}=\frac{h r^{5}}{54} \square_{4} J_{1}+3 \log \frac{r}{r_{*}} \square_{4} D_{1},
\end{gathered}
$$

where (81), (83) and (84) hold modulo terms annihilated by $d_{4}$. It is easy to see that (84) and (85) imply (80), so the latter is not independent. We thus have the five coupled equations for the five vectors $B_{1}, C_{1}, D_{1}, F_{1}$ and $J_{1}$.

\footnotetext{
${ }^{2}$ I.e. we demand that for example $\square_{4} B_{1}=m^{2} B_{1}$, but for the vectors derived from two-forms, such as $D_{1}$, we only impose the weaker condition $\square_{4} *_{4} d_{4} D_{1}=m^{2} *_{4} d_{4} D_{1}$.

${ }^{3}$ More precisely, we set $\frac{h r^{5}}{54} E_{1}=-3 \log \frac{r}{r_{*}} H_{1}=r \log \frac{r}{r_{*}} K_{1}$, and find a single second order differential equation obeyed by these fields. Thus we have found another decoupled vector, but this is not the one we are looking for. Given this relation between them, $E_{1}, H_{1}$ and $K_{1}$ do not mix with the other vectors.
} 
In the massless case, our ansatz includes the pseudoscalar found in [13]. Putting

$$
\begin{aligned}
C_{1} & =-f_{2}(r) d_{4} a(x), \\
\square_{4} D_{1} & =f_{1} d_{4} a(x), \\
B_{1}^{\prime} & =-f_{1} h r \log \frac{r}{r_{*}} d_{4} a(x), \\
F_{1} & =J_{1}=0,
\end{aligned}
$$

for some constant $f_{1}$ and a four-dimensional massless pseudoscalar $a(x)$, all equations of motion are satisfied provided

$$
\left[\frac{r^{3}}{6} f_{2}^{\prime}\right]^{\prime}-\frac{4 r}{3} f_{2}=-\frac{9}{r} f_{1} \log \frac{r}{r_{*}}
$$

in perfect agreement with the literature.

Now we would like to consider massive excitations however, and find axial vector-like solutions to the equations (80) - (85) which give rise to the superpartners of the massive scalar excitations of (44) and (45). In particular, changing variables to $W_{1}=r C_{1}$ equation (82) becomes

$$
\frac{r}{3}\left[\frac{r}{3} W_{1}^{\prime}\right]^{\prime}-W_{1}+\frac{h r^{2}}{9} \square_{4} W_{1}=\frac{2}{r} \square_{4} F_{1}-\frac{6}{h r^{2}} B_{1}^{\prime} .
$$

Thus we can identify $W_{1}$ with $\tilde{w}$ in (45), which suggests setting the right hand side of this equation proportional to the counterpart of $\tilde{z} / r$. Hence we define

$$
Z_{1} \equiv \square_{4} F_{1}-\frac{3}{h r} B_{1}^{\prime}
$$

Using (80) and (81) one can deduce that this new field obeys

$$
\frac{r}{3}\left[\frac{r}{3} Z_{1}^{\prime}\right]^{\prime}+\frac{h r^{2}}{9} \square_{4} Z_{1}=\frac{1}{r} \square_{4} W_{1}+\frac{r}{3} \square_{4}\left(J_{1}+D_{1}^{\prime}\right) .
$$

Our reduced ansatz containing five axial vectors is still too general. In order to match the spectrum of the scalar particles found above, we need to impose an additional constraint to reduce the number of dynamical vectors obeying independent second order differential equations to two. The correct constraint for our purposes is given by

$$
\square_{4}\left(J_{1}+D_{1}^{\prime}\right)=\frac{3}{r^{2}} \square_{4} W_{1} .
$$

In order to show that we can consistently impose this relation we need to examine the remaining equations. First of all, with this constraint (85) reads

$$
Z_{1}=-\frac{h r^{5}}{54} \square_{4} D_{1}^{\prime}+\frac{h r^{3}}{18} \square_{4} W_{1}+3 \log \frac{r}{r_{*}} \square_{4} D_{1} .
$$

Adding (83) and (84), using the constraint and the fact that $W_{1}$ is a mass eigenstate we find

$$
\left[\frac{h r^{3}}{18} W_{1}\right]^{\prime}+\frac{9}{r^{2}} \log \frac{r}{r_{*}} W_{1}+\frac{h^{2} r^{5}}{54} \square_{4} D_{1}=0 .
$$


Eliminating $\square_{4} D_{1}$ between the last two equations we obtain a second order differential equation containing only $W_{1}$ and $Z_{1}$. A non-trivial fact is that this equation is identical to (91). This relies heavily on the precise expression for the warp factor (150), and shows the consistency of the constraint equation with the equations of motion.

Finally, introducing a symbol for the other combination of the vectors $F_{1}$ and $B_{1}$ that appears in the equations of motion

$$
Y_{1} \equiv F_{1}^{\prime}+\frac{3}{r} B_{1}
$$

equation (84) implies

$$
\square_{4} Y_{1}=Z_{1}^{\prime}-\frac{3}{r} \square_{4} D_{1} .
$$

In summary, we have the two coupled dynamical equations

$$
\begin{aligned}
\frac{r}{3}\left[\frac{r}{3} Z_{1}^{\prime}\right]^{\prime}+\frac{h r^{2}}{9} \square_{4} Z_{1} & =\frac{2}{r} \square_{4} W_{1}, \\
\frac{r}{3}\left[\frac{r}{3} W_{1}^{\prime}\right]^{\prime}-W_{1}+\frac{h r^{2}}{9} \square_{4} W_{1} & =\frac{2}{r} Z_{1},
\end{aligned}
$$

which determine $W_{1}$ and $Z_{1}$. In terms of these $\square_{4} D_{1}$ is determined by (96), $J_{1}$ by (94), and $\square_{4} Y_{1}$ by (98). Equations (99), (100) are precisely the KT limit of the scalar equations (44), (45) up to a rescaling of the fields by a numerical factor 4

Since the KT limits of their equations of motion agree, we thus argue that the axial vectors $Z_{1}$ and $W_{1}$ are the superpartners of the coupled scalars $\tilde{z}$ and $\tilde{w}$ found above, and their massive excitations combine into vector multiplets.

\section{$5 \quad$ Effects of Compactification}

Now we will embed the KS throat into a flux compactification, along the lines of [31], and estimate the mass of the Higgs scalar. Generally, glueballs are dual to the normalizable modes localized near the bottom of the throat, and one does not expect them to be strongly affected by the bulk of the Calabi-Yau. This is indeed the case for all the massive radial excitations found in sections 2 and 3. We will see, however, that the case of the GHK scalar is more subtle and exhibits some UV sensitivity.

To model a compactification, we will introduce a UV cut-off on the radial coordinate, $\tau_{\max }$. We also need to include a deformation of the KS solution introduced by bulk effects. On the field theory side this corresponds to perturbing the Lagrangian of the cascading gauge theory by some irrelevant

\footnotetext{
${ }^{4}$ Looking at (44) one might have expected the term $2 \tilde{z} / \sinh ^{2} \tau$ to give rise to a term proportional to $Z_{1} / r^{6}$ in (99), but in fact this is not the case because it is too small to be seen in the KT limit. In the KS background it arises from a subleading term in the variation of the Ricci tensor (27) but such terms that are asymptotically suppressed by powers of $r$ compared to the leading terms are not taken into account in the KT metric. Indeed, if we write ansatz (2) in the KT background and follow the same strategy as we did for the full KS background, the term proportional to $\tilde{z} / r^{6}$ does not appear in the Einstein equations.
} 
operators. Here we are not interested in classifying all of them but rather model the compactification effects in the simplest way by considering one perturbation which simulates the main features of the compactified solution. We consider a shift of the warp factor $\delta h=$ const which corresponds to the dimension 8 operator on the field theory side [32, 33]. This also has a simple geometrical meaning: the warp factor of the compactified solution is a finite constant in the bulk of the Calabi-Yau and therefore should not drop below a certain value along the throat.

Let us introduce a small parameter $\delta$ which shifts the rescaled warp factor, $I(\tau) \rightarrow I(\tau)+\delta$, and consider the system (47)-(48) in perturbation theory near $\tilde{m}^{2}=0$ :

$$
\begin{array}{r}
\tilde{z}=\tilde{z}_{0}+\tilde{m}^{2} \tilde{z}_{1}, \\
\tilde{w}=\tilde{w}_{0}+\tilde{m}^{2} \tilde{w}_{1}, \\
\tilde{z}_{0}=\tau \operatorname{coth} \tau-1, \\
\tilde{w}_{0}(\tau)=-\frac{2^{2 / 3} 8}{9} \frac{1}{\sinh \tau} \int_{0}^{\tau} d x I(x) \sinh ^{2} x .
\end{array}
$$

At leading order in $\tilde{m}^{2}$

$$
\begin{gathered}
\tilde{z}_{1}=(\tau \operatorname{coth} \tau-1) \int_{0}^{\tau} d x u(x) \operatorname{coth} x-\operatorname{coth} \tau \int_{0}^{\tau} d x u(x)(x \operatorname{coth} x-1) \\
\tilde{w}_{1}=-\frac{1}{4 \sinh \tau} \int_{0}^{\tau} d x v(x) \frac{\sinh 2 x-2 x}{\sinh x}-\frac{\sinh 2 \tau-2 \tau}{4 \sinh \tau} \int_{\tau}^{\infty} d x v(x) \frac{1}{\sinh x} . \\
u(\tau)=-\frac{I(\tau)}{K^{2}(\tau)} \tilde{z}_{0}+\frac{9}{4 \cdot 2^{2 / 3}} K(\tau) \tilde{w}_{0}-\frac{\delta}{K^{2}} \tilde{z}_{0} \\
v(\tau)=-\frac{I(\tau)}{K^{2}(\tau)} \tilde{w}_{0}+\frac{16}{9} K(\tau) \tilde{z}_{1}-\frac{\delta}{K^{2}} \tilde{w}_{0} .
\end{gathered}
$$

Keeping in mind that for large $\tau, u \simeq-2^{-2 / 3} \delta \tau \mathrm{e}^{2 \tau / 3}$ one finds the asymptotic behavior

$$
\tilde{z}_{1}(\tau) \simeq-2^{-2 / 3} \delta \int_{0}^{\tau} d x(\tau-x) x \mathrm{e}^{2 x / 3} \simeq-\frac{9 \delta}{42^{2 / 3}} \tau \mathrm{e}^{2 \tau / 3} .
$$

This yields $v \simeq-2^{2 / 3} \delta \tau \mathrm{e}^{\tau / 3}$ and

$$
\tilde{w}_{1}=-\frac{1}{4 \sinh \tau} \int_{0}^{\tau} d x v_{0}(x) \frac{\sinh 2 x-2 x}{\sinh x}-\frac{\sinh 2 \tau-2 \tau}{4 \sinh \tau} \int_{\tau}^{\infty} d x v_{0}(x) \frac{1}{\sinh x} \simeq \frac{92^{2 / 3} \delta}{8} \tau \mathrm{e}^{\tau / 3}
$$

Finally, up the first order in the mass squared and $\delta$ :

$$
\tilde{z} \simeq \tau\left[1-\frac{9 \delta \tilde{m}^{2}}{42^{2 / 3}} \mathrm{e}^{2 \tau / 3}\right], \quad \tilde{w} \simeq-2^{4 / 3} \tau \mathrm{e}^{-\tau / 3}\left[1-\frac{9 \delta \tilde{m}^{2}}{82^{2 / 3}} \mathrm{e}^{2 \tau / 3}\right] .
$$

This suggests that for generic boundary conditions the cut-off value

$$
\tau_{\max } \simeq-\log \delta^{3 / 2} \tilde{m}^{3} .
$$

This prediction can be tested numerically. In order to do this one can specify some small $\tilde{m}$ and 


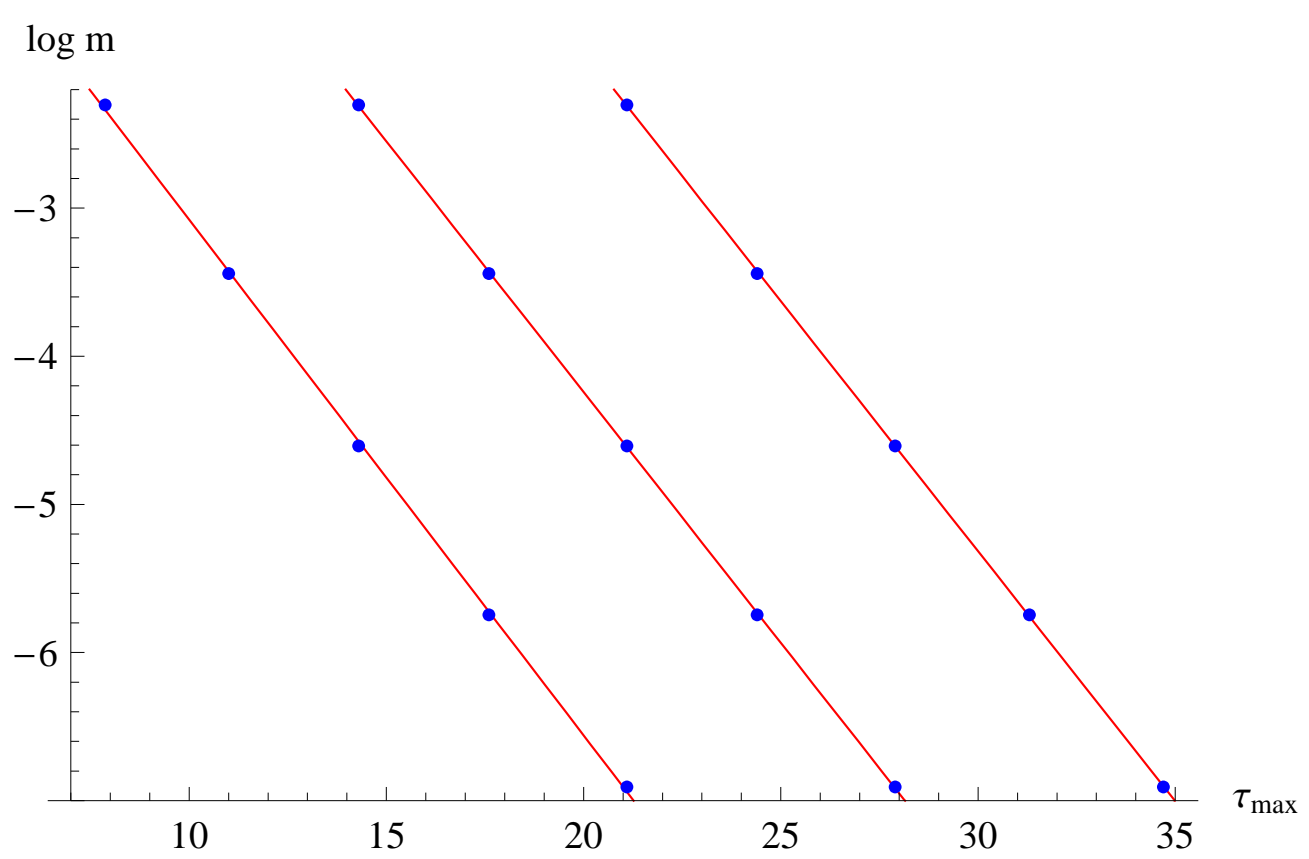

Figure 1: The dependence of $\log \tilde{m}$ on $\tau_{\max }$ is linear with the slope equal to $-1 / 3$. The three lines shown correspond to $\delta=1, \delta=0.01$ and $\delta=0.0001$.

plot the determinant

$$
\operatorname{det}\left(\begin{array}{cc}
\tilde{z}_{1}(\tau) & \tilde{z}_{2}(\tau) \\
\tilde{w}_{1}(\tau) & \tilde{w}_{2}(\tau)
\end{array}\right),
$$

of the two linearly independent solutions regular at $\tau=0$ as a function of $\tau$. The first zero marks the point $\tau_{\max }$ such that there is a regular solution with $z\left(\tau_{\max }\right)=w\left(\tau_{\max }\right)=0$. Hence $\tau_{\max }$ is the corresponding cut-off value. As Fig. 1 shows, the relation (112) holds for $\tau_{\text {max }}$ large enough that

$$
\tilde{m}^{2} \sim \delta^{-1} e^{-2 \tau_{\max } / 3}
$$

is small.

Let us consider a simple model of compactification where the throat is embedded into an asymptotically conical space that terminates at some large cut-off value $\tau_{\max }$. To calculate the mass from (114) we need to know $\delta$ as well as $\tau_{\max }$. The former is the asymptotic value of the (rescaled) warp factor. The point where the field theory warp factor approaches $\delta$ marks the UV cutoff of the field theory

$$
I\left(\tau_{U V}\right) \sim \tau_{U V} e^{-4 \tau_{U V} / 3} \simeq \delta
$$

Using this in (114) we find $\tilde{m}^{2} \sim e^{\left(4 \tau_{U V}-2 \tau_{\max }\right) / 3}$. This shows that the Higgs mass becomes parametrically small only for $\tau_{\max } \gg 2 \tau_{U V}$. This is not satisfied in general; the geometry requires only 
that $\tau_{\max }>\tau_{U V}$ because $\tau_{U V}$ is the length of the throat embedded into a CY space. With the ratio between the UV and IR scales of the field theory around $4 \cdot 10^{3}$ [24] we estimate that $\tau_{U V} \simeq 25$ 21]. The cut-off $\tau_{\max }$ can be related to the warped volume of the Calabi-Yau which, in a singular conifold approximation, is

$$
V_{6}^{w}=\operatorname{Vol}\left(T^{1,1}\right) \int_{0}^{r_{\max }} d r h(r) \sqrt{\frac{\operatorname{det} g_{6}}{\operatorname{det} g_{T^{1,1}}}},
$$

where $r \sim \epsilon^{2 / 3} e^{\tau / 3}$. The integral from zero to $r_{U V}$ is the warped volume of the throat, and from $r_{U V}$ to $r_{\max }$ is the bulk volume. Assuming that the latter dominates,

$$
V_{6}^{w} \simeq \frac{16 \pi^{3}}{27} \epsilon^{4 / 3}\left(g_{s} M \alpha^{\prime}\right)^{2}\left[r_{\max }^{6}-r_{U V}^{6}\right] r_{U V}^{-4}
$$

Requiring $\tau_{\max } \gg 50$ leads to an enormous $V_{6}^{w}$, far larger than, for example, $V_{6}^{w} \simeq 5^{6} \alpha^{\prime 3}$ in [24].

Thus, while for $\tau_{\max } \gg 2 \tau_{U V}$ the Higgs scalar becomes parametrically lighter than the other normal modes, in compactifications with realistic parameters it may actually be heavier. This is due to the special feature of its wave function $\tilde{z}$ which grows linearly with $\tau$ in the throat. The only conclusion we can draw from our simplified model of compactification is that this mode is rather UV sensitive, so to determine its mass we need to know the details of the compactification.

\section{Acknowledgements}

We thank S. Giddings, C. Herzog, J. Maldacena and M. Reece for useful discussions. This research was supported in part by the National Science Foundation under Grant No. PHY-0243680. The research of $\mathrm{AD}$ is also supported in part by Grant RFBR 07-02-00878, and Grant for Support of Scientific Schools NSh-8004.2006.2. Any opinions, findings, and conclusions or recommendations expressed in this material are those of the authors and do not necessarily reflect the views of the National Science Foundation.

\section{A The Type IIB Supergravity Equations}

Here we succinctly list the equations of motion required to study RR and NSNS 2-form perturbations. Since the dilaton and RR scalar do not enter at linear order, we set them to zero.

Bianchi identities:

$$
\begin{aligned}
& d F_{3}=0, \\
& d H_{3}=0, \\
& d F_{5}=H_{3} \wedge F_{3} .
\end{aligned}
$$


Dynamic equations:

$$
\begin{aligned}
d \star H_{3} & =-g_{s}^{2} F_{5} \wedge F_{3}, \\
d \star F_{3} & =F_{5} \wedge H_{3}, \\
F_{5} & =\star F_{5} .
\end{aligned}
$$

Einstein equation:

$$
R_{i j}=T_{i j}=\frac{g_{s}^{2}}{96} F_{i a b c d} F_{j}^{a b c d}+\frac{1}{4} H_{i a b} H_{j}^{a b}-\frac{1}{48} G_{i j} H_{a b c} H^{a b c}+\frac{g_{s}^{2}}{4} F_{i a b} F_{j}^{a b}-\frac{g_{s}^{2}}{48} G_{i j} F_{a b c} F^{a b c}
$$

\section{B Review of Warped Deformed Conifolds}

\section{B.1 The KS Solution}

The ten dimensional metric for the KS solution is

$$
d s_{10}^{2}=h(\tau)^{-1 / 2}\left(-d t^{2}+d x^{2}+d y^{2}+d z^{2}\right)+h(\tau)^{1 / 2} d s_{6}^{2},
$$

where

$$
d s_{6}^{2}=\frac{\epsilon^{4 / 3} K(\tau)}{2}\left[\frac{1}{3 K^{3}}\left(d \tau^{2}+\left(g_{5}\right)^{2}\right)+\cosh ^{2}\left(\frac{\tau}{2}\right)\left(\left(g^{3}\right)^{2}+\left(g^{4}\right)^{2}\right)+\sinh ^{2}\left(\frac{\tau}{2}\right)\left(\left(g^{1}\right)^{2}+\left(g^{2}\right)^{2}\right)\right]
$$

is the usual warped deformed conifold metric. The volume form is

$$
\text { vol }=\frac{\epsilon^{4}}{96} h^{1 / 2} \sinh ^{2} \tau d t \wedge d x^{1} \wedge d x^{2} \wedge d x^{3} \wedge d \tau \wedge g^{1} \wedge g^{2} \wedge g^{3} \wedge g^{4} \wedge g^{5}
$$

The one-forms are given in terms of angular coordinates as

$$
\begin{array}{rr}
g^{1}=\frac{e^{1}-e^{3}}{\sqrt{2}}, & g^{2}=\frac{e^{2}-e^{4}}{\sqrt{2}}, \\
g^{3}=\frac{e^{1}+e^{3}}{\sqrt{2}}, & g^{4}=\frac{e^{2}+e^{4}}{\sqrt{2}}, \\
g^{5}=e^{5}
\end{array}
$$

where

$$
\begin{gathered}
e^{1} \equiv-\sin \theta_{1} d \phi_{1}, \quad e^{2} \equiv d \theta_{1}, \\
e^{3} \equiv \cos \psi \sin \theta_{2} d \phi_{2}-\sin \psi d \theta_{2}, \\
e^{4} \equiv \sin \psi \sin \theta_{2} d \phi_{2}+\cos \psi d \theta_{2}, \\
e^{5} \equiv d \psi+\cos \theta_{1} d \phi_{1}+\cos \theta_{2} d \phi_{2}
\end{gathered}
$$


Also

$$
\begin{aligned}
& d e^{1}=-\frac{\cos \theta_{1}}{\sin \theta_{1}} e^{1} \wedge e^{2}, \\
& d e^{2}=0, \\
& d e^{3}=e^{4} \wedge e^{5}+\frac{\cos \theta_{1}}{\sin \theta_{1}} e^{4} \wedge e^{1}, \\
& d e^{4}=-e^{3} \wedge e^{5}-\frac{\cos \theta_{1}}{\sin \theta_{1}} e^{3} \wedge e^{1}, \\
& d e^{5}=-e^{1} \wedge e^{2}+e^{3} \wedge e^{4}
\end{aligned}
$$

and

$$
\begin{aligned}
& d g^{1}=\frac{1}{2}\left(g^{2}-g^{4}\right) \wedge g^{5}-\frac{1}{\sqrt{2}} \cot \theta_{1}\left(g^{1}+g^{3}\right) \wedge g^{2}, \\
& d g^{2}=-\frac{1}{2}\left(g^{1}-g^{3}\right) \wedge g^{5}-\frac{1}{\sqrt{2}} \cot \theta_{1} g^{1} \wedge g^{3}, \\
& d g^{3}=-\frac{1}{2}\left(g^{2}-g^{4}\right) \wedge g^{5}-\frac{1}{\sqrt{2}} \cot \theta_{1}\left(g^{1}+g^{3}\right) \wedge g^{4}, \\
& d g^{4}=-d g^{2}, \\
& d g^{5}=-\left(g^{1} \wedge g^{4}+g^{3} \wedge g^{2}\right) .
\end{aligned}
$$

Note that

$$
K(\tau)=\frac{(\sinh (2 \tau)-2 \tau)^{1 / 3}}{2^{1 / 3} \sinh \tau}
$$

The warp factor is

$$
h(\tau)=\left(g_{s} M \alpha^{\prime}\right)^{2} 2^{2 / 3} \varepsilon^{-8 / 3} I(\tau),
$$

where

$$
I(\tau) \equiv \int_{\tau}^{\infty} d x \frac{x \operatorname{coth} x-1}{\sinh ^{2} x}(\sinh (2 x)-2 x)^{1 / 3} .
$$

The NSNS two-form field and corresponding field strength are

$$
\begin{gathered}
B_{2}=\frac{g_{s} M \alpha^{\prime}}{2}\left[f(\tau) g^{1} \wedge g^{2}+k(\tau) g^{3} \wedge g^{4}\right] \\
H_{3}=d B_{2}=\frac{g_{s} M \alpha^{\prime}}{2}\left[d \tau \wedge\left(f^{\prime} g^{1} \wedge g^{2}+k^{\prime} g^{3} \wedge g^{4}\right)+\frac{1}{2}(k-f) g^{5} \wedge\left(g^{1} \wedge g^{3}+g^{2} \wedge g^{4}\right)\right],
\end{gathered}
$$

while the RR three-form field strength is

$$
\begin{aligned}
F_{3} & =\frac{M \alpha^{\prime}}{2}\left[g^{5} \wedge g^{3} \wedge g^{4}+d\left[F(\tau)\left(g^{1} \wedge g^{3}+g^{2} \wedge g^{4}\right)\right]\right] \\
& =\frac{M \alpha^{\prime}}{2}\left[g^{5} \wedge g^{3} \wedge g^{4}(1-F)+g^{5} \wedge g^{1} \wedge g^{2} F+F^{\prime} d \tau \wedge\left(g^{1} \wedge g^{3}+g^{2} \wedge g^{4}\right)\right]
\end{aligned}
$$


The auxiliary functions in these forms are

$$
\begin{aligned}
F(\tau) & =\frac{\sinh \tau-\tau}{2 \sinh \tau} \\
f(\tau) & =\frac{\tau \operatorname{coth} \tau-1}{2 \sinh \tau}(\cosh \tau-1) \\
k(\tau) & =\frac{\tau \operatorname{coth} \tau-1}{2 \sinh \tau}(\cosh \tau+1)
\end{aligned}
$$

Some useful identities are

$$
\begin{aligned}
k-f & =2 F^{\prime}, \\
f^{\prime} & =(1-F) \tanh ^{2}(\tau / 2), \\
k^{\prime} & =F \operatorname{coth}^{2}(\tau / 2) .
\end{aligned}
$$

The five-form field strength is given by

$$
F_{5}=(1+*) B_{2} \wedge F_{3}
$$

We also note that

$$
d g^{5}=-\left(g^{1} \wedge g^{4}+g^{3} \wedge g^{2}\right)
$$

and

$$
d g^{5} \wedge d g^{5}=-2 g^{1} \wedge g^{2} \wedge g^{3} \wedge g^{4}
$$

\section{B.2 The KT Solution}

The KT solution [8] corresponds to the large $\tau$ limit of the more general KS solution. For simplicity we take $g_{s}=\alpha^{\prime}=1, M=2$ and $N=0$. In terms of the radial coordinate $r \sim \epsilon^{2 / 3} e^{\tau / 3}$ the KT background is given by

$$
\begin{aligned}
d s^{2} & =\frac{1}{\sqrt{h}}\left(-d t^{2}+d x^{2}\right)+\sqrt{h}\left(d r^{2}+r^{2} d s_{T^{11}}^{2}\right) \\
H_{3} & =\frac{3}{r} d r \wedge \omega_{2}, \quad B_{2}=3 \log \frac{r}{r_{*}} \omega_{2}, \quad F_{3}=\omega_{3} \\
F_{5} & =(1+*) B_{2} \wedge F_{3}=3 \log \frac{r}{r_{*}}\left[\omega_{2} \wedge \omega_{3}-\frac{54}{h^{2} r^{5}} d^{4} x \wedge d r\right] .
\end{aligned}
$$

The warp factor is given by

$$
h(r)=\frac{81}{8 r^{4}}\left(1+4 \log \frac{r}{r_{*}}\right),
$$

and the conifold metric is

$$
d s_{T^{11}}^{2}=\frac{1}{9}\left(g^{5}\right)^{2}+\frac{1}{6} \sum_{i=1}^{4}\left(g^{i}\right)^{2} .
$$


The volume form is given by

$$
\mathrm{vol}=\frac{\sqrt{h} r^{5}}{54} d^{4} x \wedge \omega_{2} \wedge \omega_{3} \wedge d r
$$

Here we have introduced the two harmonic forms,

$$
\begin{gathered}
\omega_{2}=\frac{1}{2}\left(g^{1} \wedge g^{2}+g^{3} \wedge g^{4}\right)=\frac{1}{2}\left(\sin \theta_{1} d \theta_{1} \wedge d \phi_{1}-\sin \theta_{2} d \theta_{2} \wedge d \phi_{2}\right), \\
\omega_{3}=\omega_{2} \wedge g^{5} .
\end{gathered}
$$

\section{B.3 BHM Normalization}

Here we show how to find the conversion factor between the dimensionless mass squared $\tilde{m}^{2}$ and the mass in the normalization of Berg, Haack and Mück [15]. We note that the BHM conventions correspond to the KS solution with an extra relation between $\epsilon$ and $M$. The authors of [15] use the notations of the general PT ansatz (as given in Eq. (3.8) of [14]):

$$
\begin{aligned}
& d s^{2}=\mathrm{e}^{2 p-x} d s_{5}^{2}+\left(\mathrm{e}^{x+g}+a^{2} \mathrm{e}^{x-g}\right)\left(e_{1}^{2}+e_{2}^{2}\right)+\mathrm{e}^{x-g}\left[e_{3}^{2}+e_{4}^{2}-2 a\left(e_{1} e_{3}+e_{2} e_{4}\right)\right]+\mathrm{e}^{-6 p-x} e_{5}^{2}, \\
& d s_{5}^{2}=d r^{2}+\mathrm{e}^{2 A(r)} \eta_{i j} d x^{i} d x^{j} .
\end{aligned}
$$

After setting 5

$$
a=\tanh y=\frac{1}{\cosh \tau}, \quad \mathrm{e}^{-g}=\cosh y=\operatorname{coth} \tau
$$

it reduces to the KS form

$d s^{2}=\mathrm{e}^{2 A+2 p-x} \eta_{i j} d x^{i} d x^{j}+\frac{\mathrm{e}^{x}}{\sinh \tau}\left[\operatorname{coth} \tau\left(e_{1}^{2}+e_{2}^{2}+e_{3}^{2}+e_{4}^{2}\right)+\frac{2}{\sinh \tau}\left(e_{1} e_{3}+e_{2} e_{4}\right)+\mathrm{e}^{-6 p-2 x}\left(d \tau^{2}+e_{5}^{2}\right)\right]$.

The radial KS coordinate $\tau$ is introduced according to

$$
\partial_{\tau}=\mathrm{e}^{-4 p} \partial_{r}
$$

Note that in the KS notation the conifold metric (122) can be rewritten as

$$
d s_{6}^{2}=\frac{\epsilon^{4 / 3} K(\tau)}{2}\left[\frac{1}{3 K^{3}}\left(d \tau^{2}+\left(e_{5}\right)^{2}\right)+\frac{1}{2} \cosh \tau\left(e_{1}^{2}+e_{2}^{2}+e_{3}^{2}+e_{4}^{2}\right)+e_{1} e_{3}+e_{2} e_{4}\right] .
$$

In terms of $\tau$, the PT variables necessary to describe the metric for the KS background solution take the form

$$
\begin{aligned}
\Phi & =\Phi_{0}, \\
\mathrm{e}^{y} & =\tanh (\tau / 2), \\
\frac{2}{3} \mathrm{e}^{6 p+2 x} & =\operatorname{coth} \tau-\frac{\tau}{\sinh ^{2} \tau}, \\
\mathrm{e}^{2 x / 3-4 p} & =6^{-2 / 3} M^{2} \mathrm{e}^{\Phi_{0}} I(\tau) \sinh ^{4 / 3} \tau .
\end{aligned}
$$

\footnotetext{
${ }^{5}$ The Papadopoulos-Tseytlin [18] variables are $\left(x, p, y, \Phi, b, h_{1}, h_{2}\right)$.
} 
In the BHM normalization

$$
\mathrm{e}^{-2 A-8 p}=\left(\mathrm{e}^{-6 p-2 x} \sinh \tau\right)^{2 / 3} \frac{I(\tau)}{I_{0}}, \quad I_{0} \equiv I(0) .
$$

These equations give for the coefficients

$$
\begin{aligned}
\mathrm{e}^{6 p+2 x} & =\frac{3}{2} K^{3} \sinh \tau \\
\mathrm{e}^{x} & =2^{-2 / 3} \mathrm{e}^{\Phi_{0} / 2} M K(\tau) \sinh \tau \sqrt{I(\tau)}, \\
\mathrm{e}^{2 A+2 p-x} & =\sqrt{\mathrm{e}^{2 x / 3-4 p}} \sinh ^{-2 \tau / 3} \frac{I_{0}}{I}=6^{-1 / 3} \mathrm{e}^{\Phi_{0} / 2} M \frac{I_{0}}{\sqrt{I}} .
\end{aligned}
$$

Comparing these coefficients with those of the KS solution we find 6

$$
\begin{aligned}
\frac{\epsilon^{4 / 3}}{M^{2}} & =3^{-1 / 3} \mathrm{e}^{\Phi_{0} / 2} I_{0}, \\
\mathrm{e}^{\Phi_{0} / 2} & =\frac{1}{2} .
\end{aligned}
$$

This yields $\epsilon^{4 / 3} / M^{2}=3^{-1 / 3} I_{0} / 2$. Then using (46) we get for the four-dimensional mass in the BHM normalization

$$
m_{B H M}^{2}=m_{4}^{2}=\tilde{m}^{2} \frac{6}{2^{2 / 3}} \frac{\epsilon^{4 / 3}}{M^{2}}=(3 / 2)^{2 / 3} I_{0} \tilde{m}^{2} .
$$

\section{Equations of Motion for Vector Superpartners}

With the ansatz (77), the deformations of the field strengths are

$$
\begin{aligned}
& \delta H_{3}=-*_{4} \square_{4} H_{1}+\left(*_{4} d_{4} H_{1}^{\prime}+d_{4} J_{1}\right) \wedge d r \\
* & \delta H_{3}=-\frac{h^{2} r^{5}}{54} \square_{4} H_{1} \wedge \omega_{2} \wedge \omega_{3} \wedge d r+\frac{h r^{5}}{54}\left(d_{4} H_{1}^{\prime}-*_{4} d_{4} J_{1}\right) \wedge \omega_{2} \wedge \omega_{3} ; \\
\delta F_{3}= & d_{4} C_{1} \wedge g^{5}-C_{1}^{\prime} \wedge d r \wedge g^{5}-C_{1} \wedge d g^{5}+\left(*_{4} d_{4} D_{1}^{\prime}+d_{4} E_{1}\right) \wedge d r+d_{4} *_{4} d_{4} D_{1}, \\
* \delta F_{3}= & \frac{h r^{3}}{6} *_{4} d_{4} C_{1} \wedge \omega_{2} \wedge \omega_{2} \wedge d r+\frac{r^{3}}{6} *_{4} C_{1}^{\prime} \wedge \omega_{2} \wedge \omega_{2}+\frac{r}{3} *_{4} C_{1} \wedge d g^{5} \wedge g^{5} \wedge d r \\
& +\frac{h r^{5}}{54}\left(d_{4} D_{1}^{\prime}-*_{4} d_{4} E_{1}\right) \wedge \omega_{2} \wedge \omega_{3}-\frac{h^{2} r^{5}}{54} \square_{4} D_{1} \wedge \omega_{2} \wedge \omega_{3} \wedge d r ; \\
\delta F_{5}= & \delta \mathcal{F}_{5}+* \delta \mathcal{F}_{5}, \\
\delta \mathcal{F}_{5}= & \left(d_{4} B_{1}-B_{1}^{\prime} \wedge d r\right) \wedge \omega_{3}+\left(-*_{4} \square_{4} F_{1}+*{ }_{4} d_{4} F_{1}^{\prime} \wedge d r\right) \wedge \omega_{2}+d_{4} K_{1} \wedge d r \wedge \omega_{2}, \\
* \delta \mathcal{F}_{5}= & \frac{3}{r} *_{4} d_{4} B_{1} \wedge \omega_{2} \wedge d r+\frac{3}{h r} *_{4} B_{1}^{\prime} \wedge \omega_{2}-\frac{h r}{3} \square_{4} F_{1} \wedge \omega_{3} \wedge d r \\
& +\frac{r}{3}\left(d_{4} F_{1}^{\prime}-*_{4} d_{4} K_{1}\right) \wedge \omega_{3} .
\end{aligned}
$$

${ }^{6}$ We set $g_{s}=\alpha^{\prime}=1$ according to [15]. 
The equations of motion that result from this ansatz are as follows. The Bianchi identity for $F_{5}$ gives

$$
\begin{aligned}
\frac{r}{3} *_{4} \square_{4} K_{1} & =-*_{4} \square_{4} H_{1}, \\
-\frac{3}{r} *_{4} \square_{4} B_{1}-\left[\frac{3}{h r} *_{4} B_{1}^{\prime}\right]^{\prime} & =\frac{3}{r} *_{4} \square_{4} D_{1}, \\
\frac{h r}{3} d_{4} \square_{4} F_{1}+\left[\frac{r}{3}\left(d_{4} F_{1}^{\prime}-*_{4} d_{4} K_{1}\right)\right]^{\prime} & =*_{4} d_{4} H_{1}^{\prime}+d_{4} J_{1}+\frac{3}{r} d_{4} C_{1} .
\end{aligned}
$$

From the equations for $* F_{3}$ in (119) we have

$$
\begin{aligned}
\frac{h r^{5}}{54} *_{4} \square_{4} E_{1} & =-3 \log \frac{r}{r_{*}} *_{4} \square_{4} H_{1}, \\
-\frac{h r^{3}}{6} *_{4} \square_{4} C_{1}-\left[\frac{r^{3}}{6} *_{4} C_{1}^{\prime}\right]^{\prime}+\frac{4 r}{3} *_{4} C_{1}= & -\frac{3}{r} *_{4} \square_{4} F_{1}+\frac{9}{h r^{2}} *_{4} B_{1}^{\prime}, \\
{\left[\frac{h r^{5}}{54}\left(d_{4} D_{1}^{\prime}-*_{4} d_{4} E_{1}\right)\right]^{\prime}+\frac{h^{2} r^{5}}{54} d_{4} \square_{4} D_{1}=} & *_{4} d_{4} K_{1}-d_{4} F_{1}^{\prime} \\
& -3 \log \frac{r}{r_{*}}\left(*_{4} d_{4} H_{1}^{\prime}+d_{4} J_{1}\right)-\frac{3}{r} d_{4} B_{1},
\end{aligned}
$$

and from the equations for $* H_{3}$

$$
\begin{aligned}
& \frac{h r^{5}}{54} *_{4} \square_{4} J_{1}=-3 \log \frac{r}{r_{*}} *_{4} \square_{4} D_{1}+*_{4} \square_{4} F_{1}-\frac{3}{h r} *_{4} B_{1}^{\prime}, \\
& \frac{h^{2} r^{5}}{54} d_{4} \square_{4} H_{1}+\left[\frac{h r^{5}}{54}\left(d_{4} H_{1}^{\prime}-*_{4} d_{4} J_{1}\right)\right]^{\prime}=3 \log \frac{r}{r_{*}}\left(*_{4} d_{4} D_{1}^{\prime}+d_{4} E_{1}\right) \\
&-*_{4} d_{4} F_{1}^{\prime}-d_{4} K_{1}-\frac{3}{r} *_{4} d_{4} B_{1} .
\end{aligned}
$$

\section{Numerical Analysis: Finding the Spectra}

A standard method of finding the spectrum of a single second-order differential equation is the shooting technique. For a system of several coupled linear equations the shooting method has to be generalized [15]. Here we will focus on the subtleties specific to the system of equations (47) and (48). The idea of the calculation (called the determinant method [15]) is to set the initial conditions at infinity corresponding to the two solutions regular at infinity, $\left(\begin{array}{c}\tilde{z}_{1}(\tau) \\ \tilde{w}_{1}(\tau)\end{array}\right)$ and $\left(\begin{array}{c}\tilde{z}_{2}(\tau) \\ \tilde{w}_{2}(\tau)\end{array}\right)$, and extend them numerically to small $\tau$. Then the matrix

$$
\left(\begin{array}{cc}
\tilde{z}_{1}(0) & \tilde{z}_{2}(0) \\
\tilde{w}_{1}(0) & \tilde{w}_{2}(0)
\end{array}\right)
$$

becomes degenerate at the critical points (eigenvalues) in the spectral parameter space. 
Let us find the asymptotic behavior of regular and singular solutions near both zero and infinity. At small $\tau$ equations (47) and (48) decouple,

$$
\begin{gathered}
\tilde{z}^{\prime \prime}-\frac{2}{\tau^{2}} \tilde{z}=0, \\
\tilde{w}^{\prime \prime}-\frac{2}{\tau^{2}} \tilde{w}=0 .
\end{gathered}
$$

There are the two regular solutions with $\tilde{z}, \tilde{w} \sim \tau^{2}$ and the two singular solutions with $\tilde{z}, \tilde{w} \sim 1 / \tau$. For large $\tau$ we have

$$
\begin{aligned}
\tilde{z}^{\prime \prime} & =\tilde{m}^{2} \frac{9}{4 \cdot 2^{1 / 3}} \mathrm{e}^{-\tau / 3} \tilde{w}, \\
\tilde{w}^{\prime \prime}-\tilde{w} & =\frac{16 \cdot 2^{1 / 3}}{9} \mathrm{e}^{-\tau / 3} \tilde{z} .
\end{aligned}
$$

The asymptotic behavior of the two regular solutions is

$$
\left(\begin{array}{c}
\tilde{z}_{1} \\
\tilde{w}_{1}
\end{array}\right)=\left(\begin{array}{c}
1 \\
-2^{4 / 3} \mathrm{e}^{-\tau / 3}
\end{array}\right), \quad\left(\begin{array}{c}
\tilde{z}_{2} \\
\tilde{w}_{2}
\end{array}\right)=\left(\begin{array}{c}
\frac{81}{64 \cdot 2^{1 / 3}} \tilde{m}^{2} \mathrm{e}^{-4 \tau / 3} \\
\mathrm{e}^{-\tau}
\end{array}\right) ;
$$

and the singular solutions are

$$
\left(\begin{array}{c}
\tilde{z}_{3} \\
\tilde{w}_{3}
\end{array}\right)=\left(\begin{array}{c}
\tau \\
-2^{4 / 3}\left(\tau-\frac{3}{4}\right) \mathrm{e}^{-\tau / 3}
\end{array}\right), \quad\left(\begin{array}{c}
\tilde{z}_{4} \\
\tilde{w}_{4}
\end{array}\right)=\left(\begin{array}{c}
\frac{81}{16 \cdot 2^{1 / 3}} \tilde{m}^{2} \mathrm{e}^{2 \tau / 3} \\
\mathrm{e}^{\tau}
\end{array}\right) .
$$

A particular subtlety of this setup is that at large $\tau$ the two singular solutions don't diverge equally fast: one of them grows exponentially while the other is only linear in $\tau$. This makes it difficult to start shooting from zero: imposing the regularity condition at infinity would require vanishing of both linear and exponential terms. To cancel the linear term in the presence of the exponential one is difficult to do numerically. That is why for this particular system it is convenient to start shooting from large $\tau$, since both singular solutions at zero share the same behavior $(\sim 1 / \tau)$.

\section{References}

[1] I. R. Klebanov and M. J. Strassler, "Supergravity and a confining gauge theory: Duality cascades and chiSB-resolution of naked singularities," JHEP 0008, 052 (2000) arXiv:hep-th/0007191.

[2] J. M. Maldacena, "The large N limit of superconformal field theories and supergravity," Adv. Theor. Math. Phys. 2 (1998) 231 [Int. J. Theor. Phys. 38 (1999) 1113] arXiv:hep-th/9711200].

[3] S. S. Gubser, I. R. Klebanov and A. M. Polyakov, "Gauge theory correlators from non-critical string theory," Phys. Lett. B 428, 105 (1998) arXiv:hep-th/9802109.

[4] E. Witten, "Anti-de Sitter space and holography," Adv. Theor. Math. Phys. 2, 253 (1998) arXiv:hep-th/9802150. 
[5] I. R. Klebanov and E. Witten, "Superconformal field theory on threebranes at a Calabi-Yau singularity," Nucl. Phys. B 536, 199 (1998) arXiv:hep-th/9807080.

[6] S. S. Gubser and I. R. Klebanov, "Baryons and domain walls in an N = 1 superconformal gauge theory," Phys. Rev. D 58, 125025 (1998) arXiv:hep-th/9808075.

[7] I. R. Klebanov and N. A. Nekrasov, "Gravity duals of fractional branes and logarithmic RG flow," Nucl. Phys. B 574, 263 (2000) arXiv:hep-th/9911096.

[8] I. R. Klebanov and A. A. Tseytlin, "Gravity duals of supersymmetric SU(N) x SU(N+M) gauge theories," Nucl. Phys. B 578, 123 (2000) arXiv:hep-th/0002159].

[9] C. P. Herzog, I. R. Klebanov and P. Ouyang, "Remarks on the warped deformed conifold," arXiv:hep-th/0108101; "D-branes on the conifold and N = 1 gauge / gravity dualities," arXiv:hep-th/0205100.

[10] M. J. Strassler, "The duality cascade," arXiv:hep-th/0505153.

[11] M. Krasnitz, "A two point function in a cascading $N=1$ gauge theory from supergravity," arXiv:hep-th/0011179; "Correlation functions in a cascading $\mathrm{N}=1$ gauge theory from supergravity," JHEP 0212, 048 (2002) arXiv:hep-th/0209163.

[12] E. Caceres, "A Brief Review Of Glueball Masses From Gauge / Gravity Duality," J. Phys. Conf. Ser. 24, 111 (2005).

[13] S. S. Gubser, C. P. Herzog and I. R. Klebanov, "Symmetry breaking and axionic strings in the warped deformed conifold," JHEP 0409, 036 (2004) arXiv:hep-th/0405282; "Variations on the warped deformed conifold," Comptes Rendus Physique 5 (2004) 1031 arXiv:hep-th/0409186].

[14] M. Berg, M. Haack and W. Muck, "Bulk dynamics in confining gauge theories," Nucl. Phys. B 736, 82 (2006) arXiv:hep-th/0507285.

[15] M. Berg, M. Haack and W. Muck, "Glueballs vs. gluinoballs: Fluctuation spectra in nonAdS/non-CFT," arXiv:hep-th/0612224.

[16] R. Argurio, G. Ferretti and C. Petersson, "Massless fermionic bound states and the gauge/gravity correspondence," JHEP 0603, 043 (2006) arXiv:hep-th/0601180].

[17] A. Dymarsky and D. Melnikov, "Gravity Multiplet on KS and BB Backgrounds," arXiv:0710.4517 [hep-th].

[18] G. Papadopoulos and A. A. Tseytlin, "Complex geometry of conifolds and 5-brane wrapped on 2-sphere," Class. Quant. Grav. 18, 1333 (2001) arXiv:hep-th/0012034.

[19] O. Aharony, "A note on the holographic interpretation of string theory backgrounds with varying flux," JHEP 0103, 012 (2001) arXiv:hep-th/0101013.

[20] A. Butti, M. Grana, R. Minasian, M. Petrini and A. Zaffaroni, "The baryonic branch of Klebanov-Strassler solution: A supersymmetric family of SU(3) structure backgrounds," JHEP 0503, 069 (2005) arXiv:hep-th/0412187. 
[21] A. Dymarsky, I. R. Klebanov and N. Seiberg, "On the moduli space of the cascading SU(M+p) x SU(p) gauge theory," JHEP 0601, 155 (2006) arXiv:hep-th/0511254.

[22] M. K. Benna, A. Dymarsky and I. R. Klebanov, "Baryonic condensates on the conifold," JHEP 0708, 034 (2007) arXiv:hep-th/0612136.

[23] S. Kachru, R. Kallosh, A. Linde and S. P. Trivedi, "De Sitter vacua in string theory," Phys. Rev. D 68, 046005 (2003) arXiv:hep-th/0301240.

[24] S. Kachru, R. Kallosh, A. Linde, J. Maldacena, L. McAllister and S. P. Trivedi, "Towards inflation in string theory," JCAP 0310, 013 (2003) arXiv:hep-th/0308055].

[25] D. Baumann, A. Dymarsky, I. R. Klebanov, L. McAllister and P. J. Steinhardt, "A Delicate Universe," Phys. Rev. Lett. 99, 141601 (2007) [arXiv:0705.3837 [hep-th]]; D. Baumann, A. Dymarsky, I. R. Klebanov and L. McAllister, "Towards an Explicit Model of D-brane Inflation," arXiv:0706.0360 [hep-th].

[26] N. Barnaby and J. M. Cline, "Tachyon defect formation and reheating in brane-antibrane inflation," Int. J. Mod. Phys. A 19, 5455 (2004) arXiv:hep-th/0410030; N. Barnaby, C. P. Burgess and J. M. Cline, "Warped reheating in brane-antibrane inflation," JCAP 0504, 007 (2005) arXiv:hep-th/0412040.

[27] L. Kofman and P. Yi, "Reheating the universe after string theory inflation," Phys. Rev. D 72, 106001 (2005) arXiv:hep-th/0507257.

[28] A. R. Frey, A. Mazumdar and R. Myers, "Stringy effects during inflation and reheating," Phys. Rev. D 73, 026003 (2006) arXiv:hep-th/0508139.

[29] A. Karch, E. Katz, D. T. Son and M. A. Stephanov, "Linear confinement and AdS/QCD," Phys. Rev. D 74, 015005 (2006) arXiv:hep-ph/0602229.

[30] M. Krasnitz, Ph.D. Thesis, Princeton University (2003).

[31] S. B. Giddings, S. Kachru and J. Polchinski, "Hierarchies from fluxes in string compactifications," Phys. Rev. D 66, 106006 (2002) arXiv:hep-th/0105097.

[32] S. S. Gubser, A. Hashimoto, I. R. Klebanov and M. Krasnitz, "Scalar absorption and the breaking of the world volume conformal invariance," Nucl. Phys. B 526, 393 (1998) arXiv:hep-th/9803023; S. S. Gubser and A. Hashimoto, "Exact absorption probabilities for the D3-brane," Commun. Math. Phys. 203, 325 (1999) arXiv:hep-th/9805140.

[33] S. Kachru, L. McAllister and R. Sundrum, "Sequestering in string theory," JHEP 0710, 013 (2007) arXiv:hep-th/0703105. 